\title{
Exploration of Nonlinear Optical Enhancement and Interesting Optical Behavior with Pyrene Moiety as the Conjugated Donor and Efficient Modification in Acceptor Moieties
}

\section{Muhammad Khalid ( $\nabla$ khalid@iq.usp.br)}

KFUEIT: Khwaja Farid University of Engineering and Information Technology https://orcid.org/00000002-1899-5689

\section{Muhammad Usman Khan}

University of Okara

Nimra Azhar

Khwaja Fareed University of Engineering \& Information Technology Faculty of Engineering and Technology

\section{Muhammad Nadeem Arshad}

King Abdulaziz University

\section{Abdullah M. Asiri}

King Abdulaziz University

\section{Ataualpa Albert Carmo Braga}

University of Sao Paulo: Universidade de Sao Paulo

\section{Muhammad Nadeem Akhtar}

Islamia University: The Islamia University of Bahawalpur Pakistan

\section{Research Article}

Keywords: Linear polarizability, First hyperpolarizability, Second hyperpolarizability, UV-Vis, FMO, Endcapped moieties, non-fullerene acceptors.

Posted Date: September 20th, 2021

DOl: https://doi.org/10.21203/rs.3.rs-833929/v1

License: @ (i) This work is licensed under a Creative Commons Attribution 4.0 International License. Read Full License 
Exploration of Nonlinear Optical Enhancement and Interesting Optical Behavior with Pyrene Moiety as the Conjugated Donor and Efficient Modification in Acceptor Moieties

Muhammad Khalid, *1 ${ }^{1}$ Muhammad Usman Khan, ${ }^{2}$ Nimra Azhar, ${ }^{1}$ Muhammad Nadeem Arshad $^{3,4}$, Abdullah M. Asiri ${ }^{3,4}$, Ataualpa Albert Carmo Braga, ${ }^{5}$ Muhammad Nadeem Akhtar ${ }^{6}$

${ }^{1}$ Department of Chemistry, Khwaja Fareed University of Engineering \& Information Technology, Rahim Yar Khan, 64200, Pakistan

${ }^{2}$ Department of Chemistry, University of Okara, Okara-56300, Pakistan

${ }^{3}$ Chemistry Department, Faculty of Science, King Abdulaziz University, Jeddah 21589, P.O. Box 80203, Saudi Arabia.

${ }^{4}$ Center of Excellence for Advanced Material Research (CEAMR), King Abdulaziz University, Jeddah 21589, P.O. Box 80203, Saudi Arabia

${ }^{5}$ Departamento de Química Fundamental, Instituto de Química, Universidade de São Paulo, Av. Prof. Lineu Prestes, 748, São Paulo, 05508-000, Brazil.

${ }^{6}$ Department of Chemistry, Baghdad-ul-Jadeed Campus, The Islamia University of Bahawalpur, Bahawalpur, 63100, Pakistan.

*Corresponding authors E-mail addresses:

Dr. Muhammad Khalid (muhammad.khalid@kfueit.edu.pk; Khalid@iq.usp.br)

\section{Abstract}

Herein, a series of new pyrene based hexylcyanoacetate derivatives (HPPC1-HPPC8) with $\mathrm{A}-\pi-\mathrm{D}-\pi-\mathrm{D}$ configuration were designed by end-capped modeling of non-fullerene acceptors on the structure of reference compound named dihexyl 3,3'-(pyrene-1,6-diylbis(4,1phenylene))(2E,2'E)-bis(2-cyanoacrylate) HPPCR. Quantum chemical calculations of HPPCR and HPPC1-HPPC8 were accomplished at M06/6-31G(d, p) level. The stability of molecules due to the strongest hyper conjugative interactions in HPPCR and HPPC1-HPPC8 was estimated through NBO study. Interestingly, HOMO-LUMO band-gap of HPPC1-HPPC8 was found smaller than HPPCR which resulted in large NLO response. Among all the investigated compounds HPPC7 showed the larger NLO response due to the presence of four cyanide (CN) groups which strengthens the bridge conjugation, and its band gap was found to be $2.11 \mathrm{eV}$, smaller as compared to band gap of HPPCR $(3.225 \mathrm{eV})$. The absorption spectra of HPPC1HPPC8 compounds showed maximum absorption wavelengths (483-707 $\mathrm{nm}$ ) than HPPCR (471.764nm). The designed compounds showed high NLO response than HPPCR. Amazingly, 
highest amplitude of linear polarizability $\langle\alpha\rangle$, first hyperpolarizability $\left(\beta_{\text {total }}\right)$ and second hyperpolarizability $\langle\gamma\rangle$ for HPPC7 were achieved to be 1331.191, 200112.2 and $4.131 \times 10^{7}$ (a.u), respectively. NLO response showed that the HPPC1-HPPC8 might be potential candidates for NLO applications.

Keywords: Linear polarizability; First hyperpolarizability; Second hyperpolarizability; UV-Vis; FMO; End-capped moieties; non-fullerene acceptors.

\section{Introduction}

In the recent era, it has become essential to focus our attention from use of electrons to photons for the usage of ultra-fast technology. Therefore, broad-spectrum investigations have been made in several technological grounds such as telecommunication, optical switching, data storage and optical computing [1]. Nowadays, photons are considered with great preference for the transfer of information [2-6]. The non-linear optics (NLO) have attracted significant interest, especially during the last era due to the progression in experimental communities and molecular modelling. Molecules with large optical non-linearities have gained notable significance [7-13]. Thus, wide-ranging research efforts have been made at designing the more efficient photonmanipulating materials. As a result, different attempts are being made to model innovative NLO materials have increased rapidly [14]. Organic NLO materials are preferred over the inorganic NLO materials due to their lower production costs, smaller dielectric constants, fast NLO response, higher second and third order hyperpolarizabilities and design flexibility $[11,15,16]$. The NLO properties of organic conjugated materials can be efficiently enhanced by structural tailoring and doped with fullerene acceptors [17]. Fullerene derivatives with greater photoinduced electron transfer and suitable charge separation property have been extensively used as electron acceptors for many years [18]. Unfortunately, there are certain intrinsic drawbacks of fullerene acceptors for example poor accepting power, weak light absorption in the visible range, and limited guideline on their molecular energy levels [19]. Compared with fullerene derivatives, non-fullerene acceptors (NFAs) have exclusive benefits, such as transparency, easily-tuned energy levels, efficient light absorption, easy in fabrication, diverse chemical structures and flexibility [20-24].

Literature study reveals that organic NFAs are used in organic solar cells but their usage in NLO materials has not been reported yet. We identified a compound HPPCR from literature [25] as reference compound and to the best of our knowledge, no systematic NLO study of 
HPPCR has been published yet. HPPCR is non-fullerene based (A- $\pi$-D- $\pi-A$ ) type compound which comprises of hexyl 2-cyanoacrylate as first and second acceptor, phenyl as first and second $\pi$-linker and pyrene core as a donor moiety. The literature survey revealed different types of donor acceptor moieties including donor-acceptor, donor- $\pi$-acceptor, donor- $\pi$ - $\pi$-acceptor, donor-acceptor- $\pi$-acceptor, donor-donor- $\pi$-acceptor, donor- $\pi$-acceptor- $\pi$-donor and acceptor- $\pi$ donor- $\pi$ - donor [26-29]. Frequently, a push-pull model is used for designing of A- $\pi$-D- $\pi$-D type organic compounds. The NLO compounds with push pull schemes have drawn abundant attention for researchers because of remarkable NLO results [30, 31].

In the current study, we considered HPPCR as prototype and designed eight compounds (HPPC1-HPPC8) with $\mathrm{A}-\pi-\mathrm{D}-\pi-\mathrm{D}$ architecture by structural tailoring with various halogenated non-fullerene-based acceptors. The donor and $\pi$-linker are kept preserved throughout the designing while acceptor groups are modulated. Different parameters, such as electronic properties, natural bond orbital (NBO) analysis, maximum absorption, second and third order NLO, linear polarizability and dipole moment for all investigated compounds (HPPC1-HPPC8) and the reference compound HPPCR were computed to estimate the performance of the new engineered compounds as effective NLO materials. These findings not only provide information for the designing of $A-\pi-D-\pi-D$ configuration-based novel non fullerene organic entities, but also trigger experimental researchers for the synthesis of these molecules with exceptional NLO response properties.

\section{Computational procedure}

All the calculations regarding computational analyses were carried out using Gaussian 16 program package [32]. The computations input files were developed using the Gauss View 6.0 program [33]. Density functional theory (DFT) was performed for the geometrical optimization without symmetry restrictions of reference HPPCR molecule and HPPC1-HPPC8. The DFT/M06/6- 31G (d,p) level of theory was carried out to investigate NBO and NLO properties. TD-DFT with aforesaid level was performed to investigate FMO and UV-Vis spectra of pyrene based compounds (HPPC1-HPPC8). The solvent (DCM) effect was calculated by means of conductor-like polarizable continuum (CPCM) model in all computational analyses [34]. Gaussian output file provides six linear polarizability tensors $\left(\alpha_{\mathrm{xx}}, \alpha_{\mathrm{yy}}, \alpha_{\mathrm{zz}}, \alpha_{\mathrm{xy}}, \alpha_{\mathrm{xz}}, \alpha_{\mathrm{yz}}\right)$ and ten hyperpolarizability tensors $\left(\beta_{\mathrm{xxx}}, \beta_{\mathrm{xyy}}, \beta_{\mathrm{xzz}}, \beta_{\mathrm{yyy}}, \beta_{\mathrm{xxy}}, \beta_{\mathrm{yzz}}, \beta_{\mathrm{zzz}}, \beta_{\mathrm{xxz}}, \beta_{\mathrm{yyz}}, \beta_{\mathrm{xyz}}\right)$ along x, y and z 
directions, respectively. For interpretation of results from output files, Avogadro [35] and Chemcraft [36] were employed.

Dipole moment was determined by using Eq1 [37].

$$
\mu=\left(\mu_{x}^{2}+\mu_{y}^{2}+\mu_{z}^{2}\right)^{1 / 2}
$$

Average polarizability $<\alpha>$ determined with Eq2 [34].

$$
<\alpha>=1 / 3\left(\alpha_{x x}+\alpha_{y y}+\alpha_{z z}\right)
$$

The magnitude of total first hyperpolarizability $\left(\beta_{\mathrm{tot}}\right)$ was calculated via Eq3 [34].

$$
\beta_{\mathrm{total}}=\left[\left(\beta_{\mathrm{xxx}}+\beta_{\mathrm{xyy}}+\beta_{x z z}\right)^{2}+\left(\beta_{\mathrm{yyy}}+\beta_{\mathrm{yzz}}+\beta_{\mathrm{yxx}}\right)^{2}+\left(\beta_{\mathrm{zzz}}+\beta_{\mathrm{zxx}}+\beta_{x y z}\right)^{2}\right]^{1 / 2}
$$

The second hyperpolarizability was determined by using the Eq4 [37].

$$
<\gamma>=1 / 5\left[\gamma_{x x x x}+\gamma_{y y y y}+\gamma_{z z z z}+2\left(\gamma_{x x x x}+\gamma_{y y y y}+\gamma_{z z z z}\right)\right]
$$

\section{Results and discussion}

In the present work, the experimentally synthesized compound is taken as the reference HPPCR. The reference HPPCR compound consists on A- $\pi-D-\pi-A$ configuration as shown in Figure 1. The pyrene ring in reference compound is acting as a donor moiety which has electronic donating ability. Whereas hexylcyanoacetate parts located at both ends consisting of electron withdrawing capability regarded as acceptors. The pyrene ring and hexylcyanoacetate parts are coupled via phenyl $\pi$-linker. We exchanged the first acceptor moiety of the HPPCR compound with various halogenated non-fullerene acceptors and second acceptor with the pyrene donor and also incorporated the thienyl with the second $\pi$-linker. By carrying out these modifications in the reference (HPPCR) compound, we designed eight derivatives (HPPC1HPPC8) have $A-\pi-D-\pi-D$ architecture as shown in Figure 2. The aim of our current investigation is to design the innovative non-fullerene-based acceptor materials with notable optoelectronic properties. The detailed computations were achieved to reveal that how $\pi$-linkers and numerous accepters affect the HOMO/LUMO band gaps, ICT, absorption spectra, electronic properties, linear polarizability $\langle\alpha\rangle$, first hyperpolarizability $(\beta)$ and second hyperpolarizability $\langle\gamma\rangle$ as well as NBO investigation. 


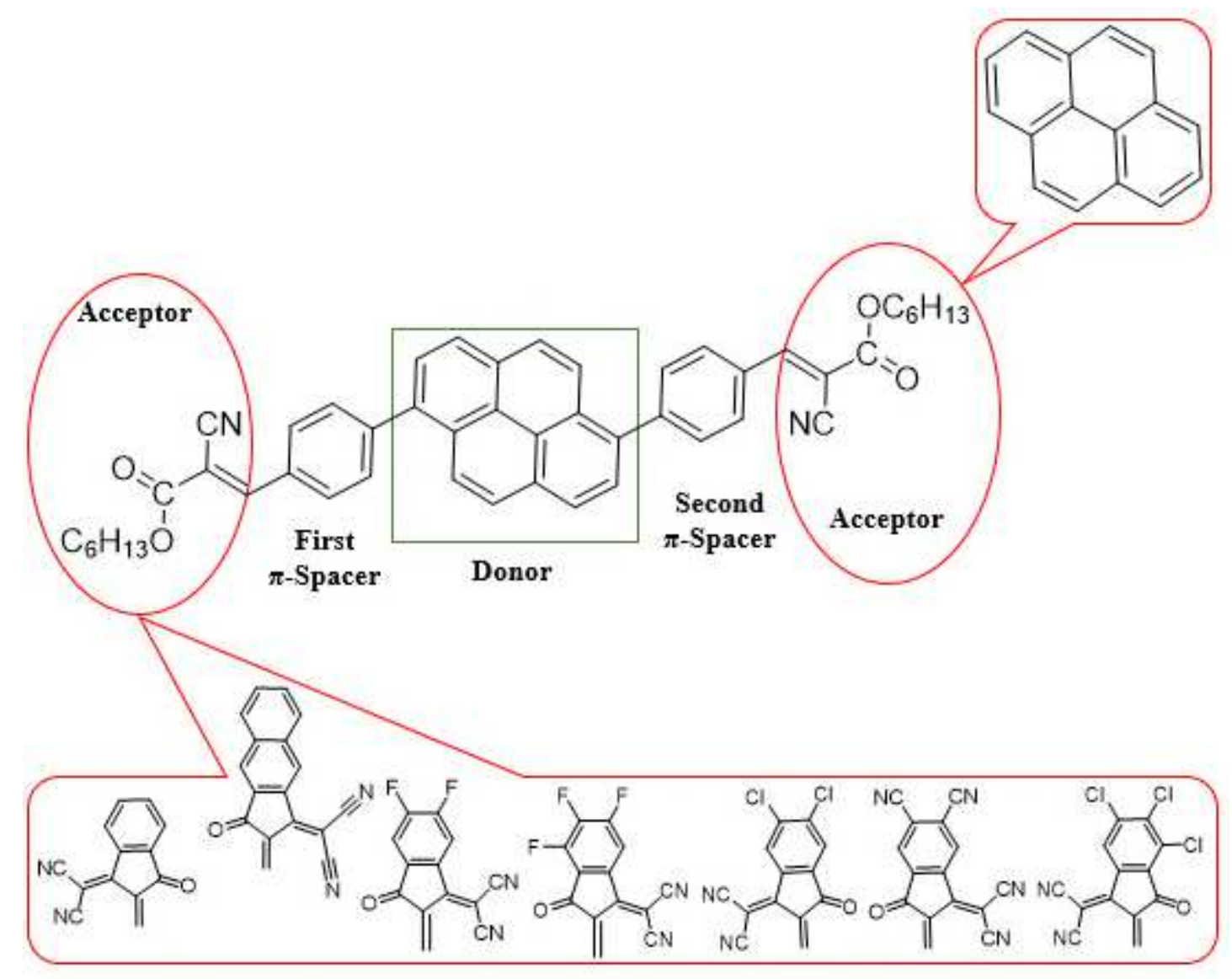

Figure 1: The sketch map of designed compounds (HPPC1-HPPC8).

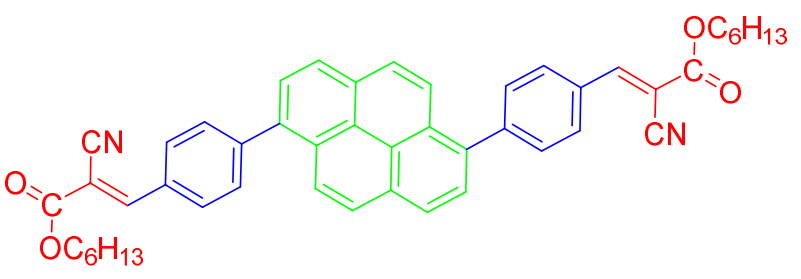

HPPCR

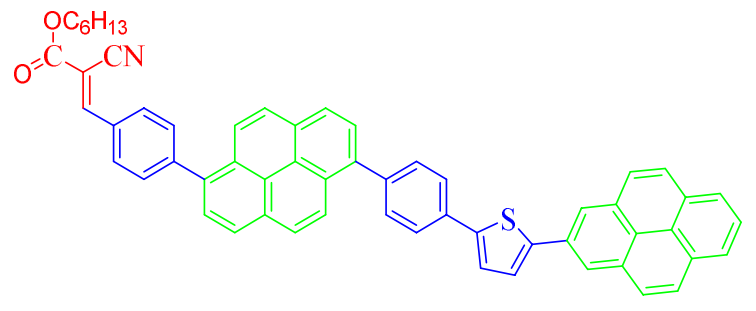

HPPC1

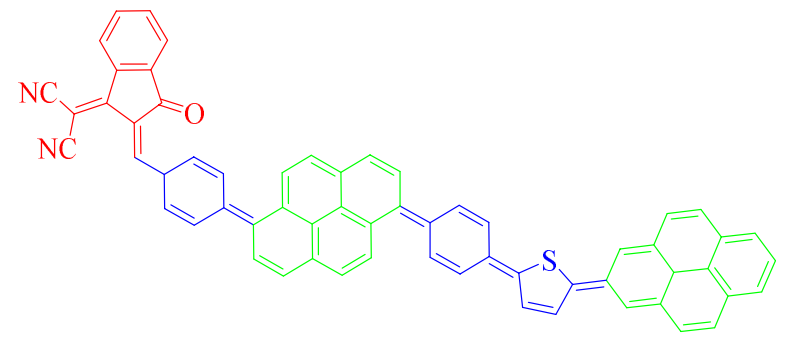

HPPC2 


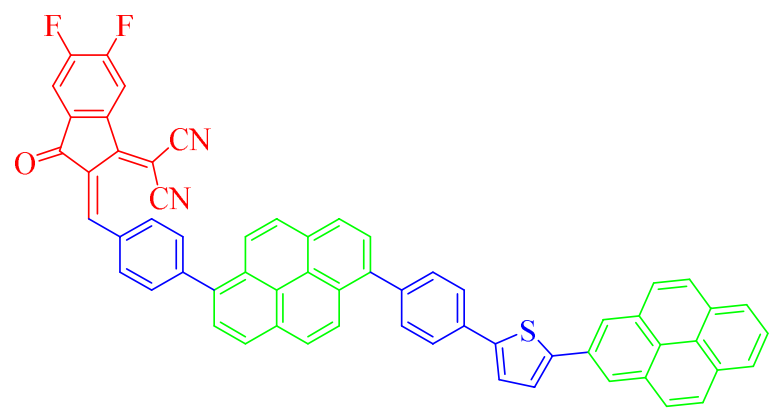

HPPC3

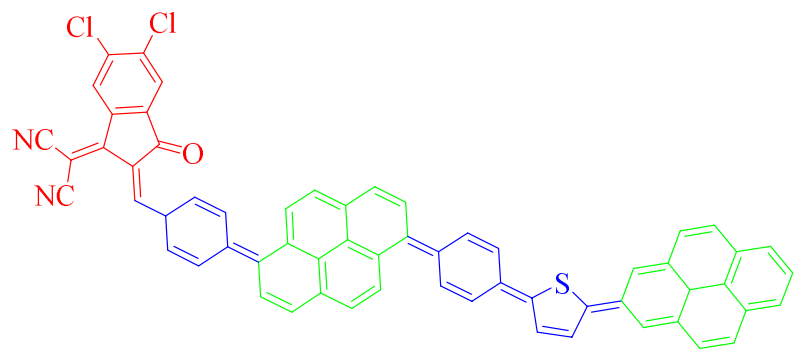

HPPC5

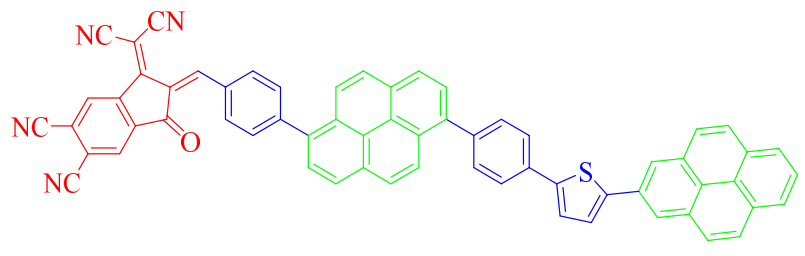

HPPC7

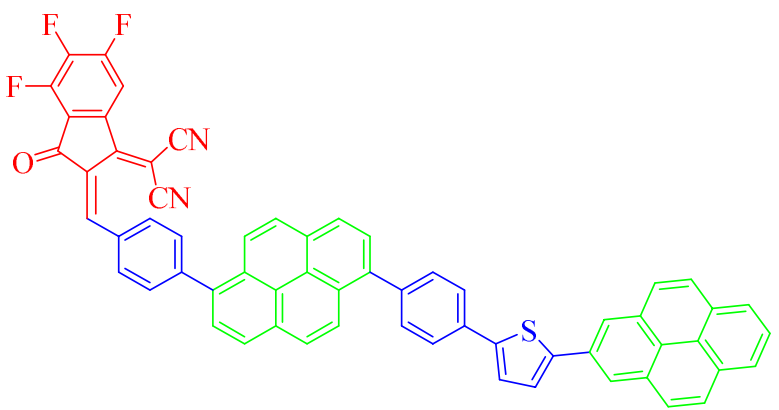

HPPC4

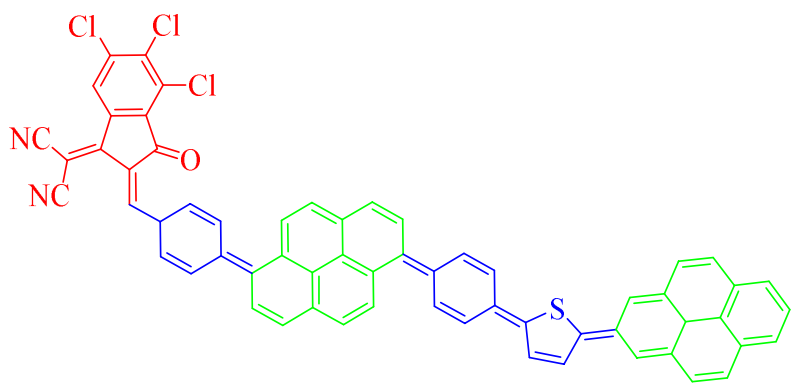

HPPC6

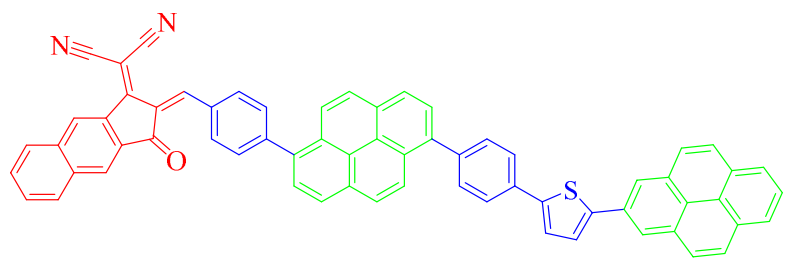

HPPC8

Acceptor $\pi$-Spacers Donor

Figure 2: Structure of (HPPCR) and designed compounds (HPPC1-HPPC8). 


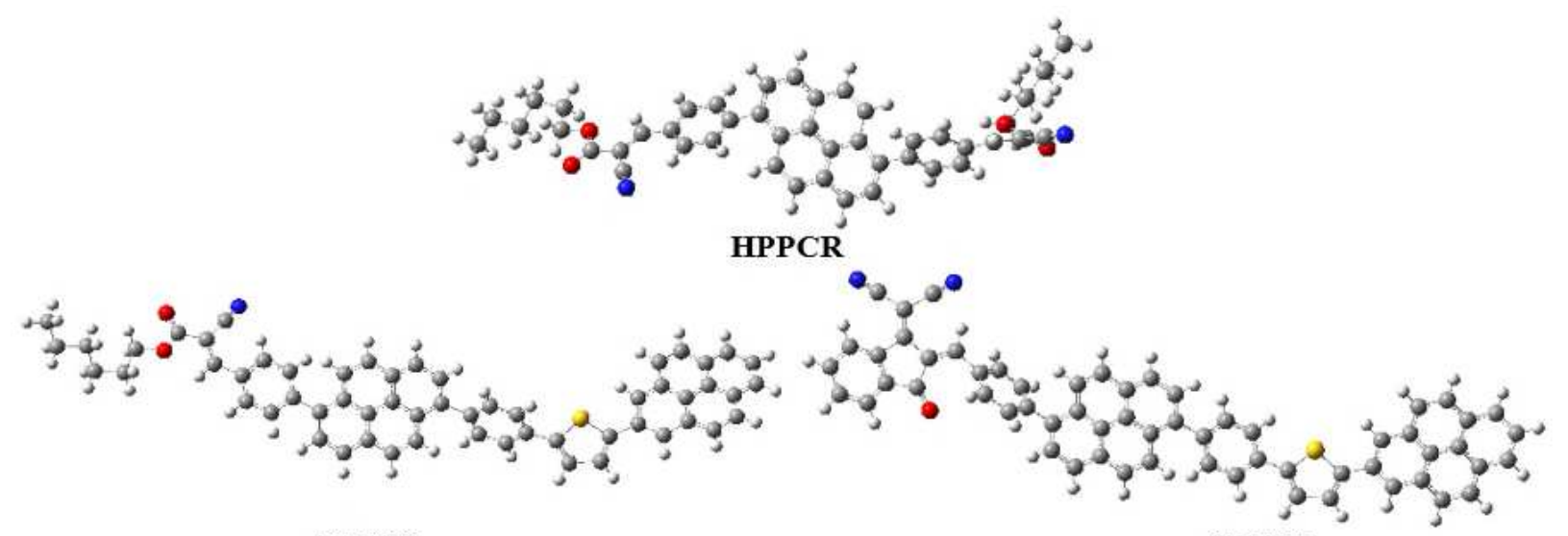

HPPC1

HPPC2

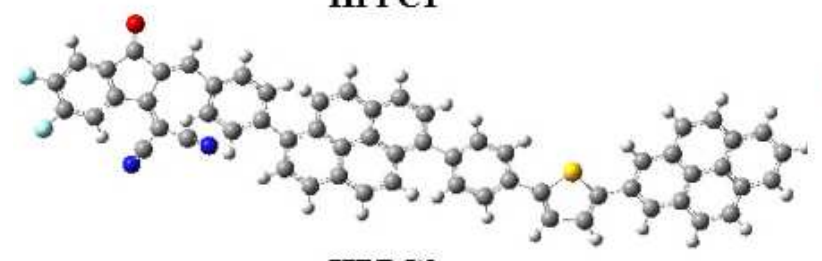

HPPC3
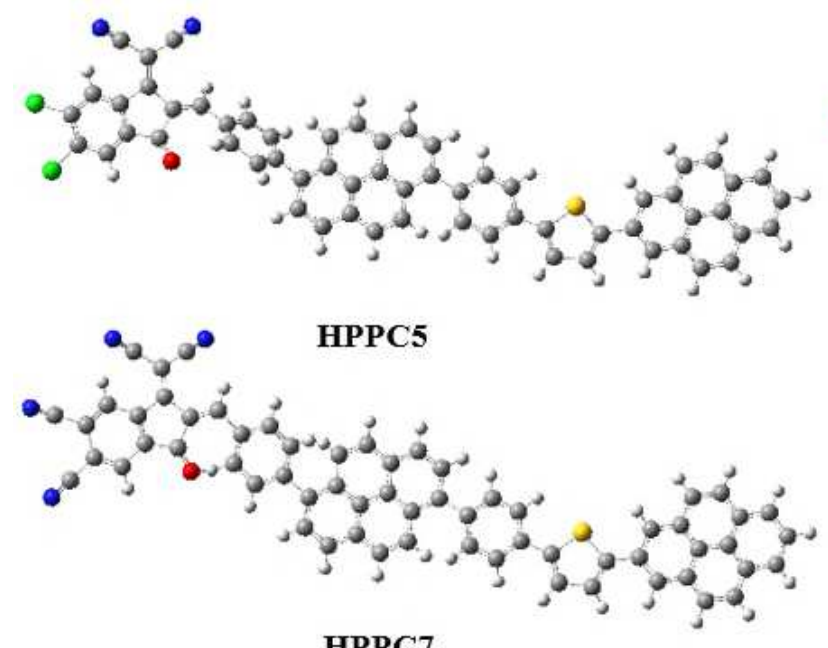

HPPC7

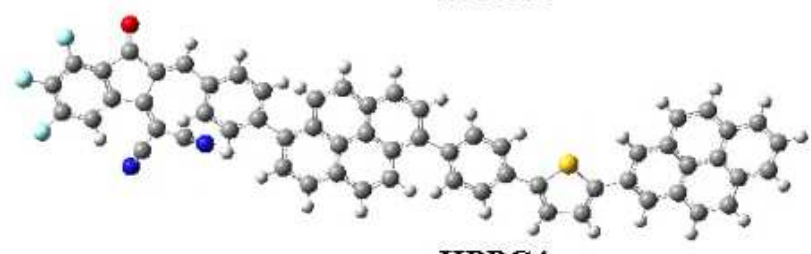

HPPC4
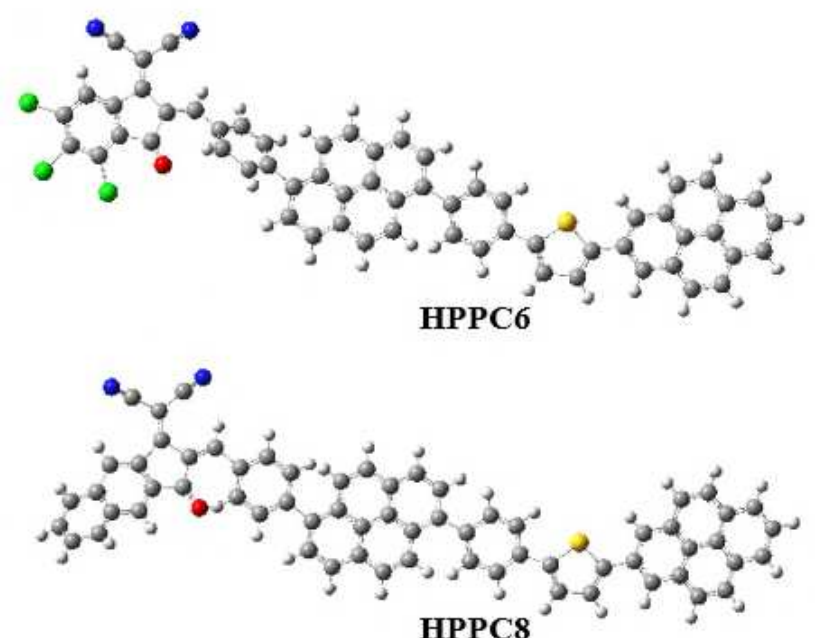

HPPC8

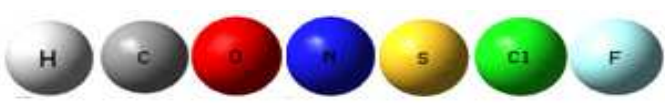

Figure 3: Optimized structures of reference compound (HPPCR) and designed compounds (HPPC1-

\section{HPPC8)}

\section{Electronic structures}

The frontier molecular orbital (FMO) plays an important role in the UV-Vis spectra, kinetic stability, chemical reactions, optical and electronic properties of the molecule [38, 39]. The highest occupied molecular orbital (HOMO), lowest unoccupied molecular orbital (LUMO) and their energy difference are essential pointers which can be used to adjust and enhance the NLO properties of the molecule [40]. The band gap $\left(\mathrm{E}_{\mathrm{g}}\right)$ is a quite essential parameter to analyze 
the softness, hardness and internal charge transfer (ICT) from the end-capped electron donor moiety towards the electron-acceptor groups via $\pi$-conjugated linker of the molecules [39, 41, 42]. The molecules having larger band gap are known as hard while with the smaller band gap are called soft molecules. Hard molecules are less reactive, stable and having a lower polarizability [43]. On the other hand, soft molecules are highly reactive, unstable and more polarizable because they need less energy for excitation [44]. A smaller energy gap leads to promising NLO response. Thus, the FMO band gap can be used for qualitative estimation of the NLO behavior of the molecules [45]. The FMO band gap of reference HPPCR and designed compounds HPPC1-HPPC8 are tabulated in the Table 1.

Table 1: Computed energies of $E_{\text {LumO, }} \mathrm{E}_{\mathrm{HOMO}}$ and band gap of studied compounds

\begin{tabular}{cccc}
\hline Compounds & $\boldsymbol{E}_{\text {Hомо }}$ & $\boldsymbol{E}_{\text {LUMO }}$ & Band Gap \\
\hline HPPCR & -5.755 & -2.530 & 3.225 \\
HPPC1 & -5.593 & -2.495 & 3.098 \\
HPPC2 & -5.595 & -3.084 & 2.511 \\
HPPC3 & -5.560 & -3.123 & 2.437 \\
HPPC4 & -5.563 & -3.196 & 2.367 \\
HPPC5 & -5.608 & -3.233 & 2.375 \\
HPPC6 & -5.597 & -3.310 & 2.287 \\
HPPC7 & -5.647 & -3.529 & 2.118 \\
HPPC8 & -5.590 & -3.146 & 2.444 \\
\hline
\end{tabular}

Units in $e V$

Table 1 shows the HOMO/LUMO calculated values of reference compound is -5.755/$2.530 \mathrm{eV}$, which is very close to experimentally determined values $-5.82 / 2.86 \mathrm{eV}$ [25] respectively. These results indicated that the implemented computational procedure was suitable to investigate HPPC1-HPPC8 compounds. All the investigated HPPC1-HPPC8 compounds exhibited smaller HOMO and LUMO values in the range of 2.118-3.098 eV as compared to HPPCR (3.225 $\mathrm{eV}$ ) which might be owing to the extended conjugation factor. This energy gap value decreased to $3.098 \mathrm{eV}$ in HPPC1 because of the replacement of second acceptor with pyrene donor, along with the incorporation of thienyl with second linker, which enhances the electron donating ability of donor group towards the acceptor group by creating strong push pull mechanism. This energy gap is further reduced in HPPC2 due to the substitution of nonfullerene acceptor group 2-(2-methylene-3-oxo-2,3-dihydro-1H-inden-1-ylidene)malononitrile. The structure of HPPC3 is designed by substitution of two fluorine groups on the acceptor unit in HPPC2 derivative. The band gap of HPPC3 is lowered owing to the two F groups 
substituents which enhances the electron withdrawing nature of acceptor moiety. The HPPC4 and HPPC5 have a comparable HOMO and LUMO energies as -5.563/-3.196 and -5.608/-3.233 $e V$, respectively. The structure of HPPC4 is designed by incorporating the F group in the acceptor moiety of HPPC3 derivative. Among all the halogens F is highly electronegative and it increases the electron withdrawing capacity of acceptor group which might be the reason for the reduction in the energy gap value $2.367 \mathrm{eV}$ in HPPC4. The structure of HPPC5 is designed by replacing the three $\mathrm{F}$ groups with the two $(-\mathrm{Cl})$ groups at the end capped acceptor moiety of HPPC4 derivative. The HPPC5 shows little bit more energy gap than HPPC4 because (-Cl) is less electronegative than (-F). The structure of HPPC6 is designed by substitution of (-Cl) group at the acceptor region of HPPC5 which might be the reason for lowering the band gap. A much smaller HOMO/LUMO energy gap was observed at $2.118 \mathrm{eV}$ in HPPC7. In fact, HPPC7 structure is designed by the replacing three $(-\mathrm{Cl})$ group with the two cyano $(-\mathrm{CN})$ groups on the acceptor unit in HPPC6 derivative. In HPPC7 the energy gap is reduced due to the substitution of cyano $(-\mathrm{CN})$ groups on the acceptor region having a larger $(-\mathrm{I})$ effect than $(-\mathrm{Cl})$ groups. These $(-\mathrm{CN})$ groups have excellent electron withdrawing nature and can withdraw more electrons toward the acceptor region. Thus, this factor intensifies the charge transfer and lowers its band gap. This is the lowest value of Egap among all the designed molecules. In the same way the HPPC8 is reported with a little bit more energy gap $\left(\mathrm{E}_{\mathrm{gap}}=2.444 \mathrm{eV}\right)$ due the effect of acceptor group 2-(2-methylene-3-oxo-2,3-dihydro-1H-cyclopenta[b]naphthalen-1-ylidene) malononitrile. Therefore, the HOMO-LUMO energy gap was remarkably reduced in all the designed molecules. The band gap increasing order: HPPC7 <HPPC6 <HPPC4 <HPPC5 <HPPC3 $<$ HPPC8 < HPPC2 <HPPC1 <HPPCR. This order shows that the incorporating various electronegative substituent in the designed compounds would be outstanding aspect to reduce the band gap values, hence, significant the NLO response [46].

In the HPPCR and HPPC1-HPPC8 charge distribution pattern depicted in the Figure 4. Such electron density distribution pattern is advantageous for efficient charge transfer phenomena. The charge transfer phenomena proved that investigated compounds would be the outstanding NLO material [45]. In the reference compound HPPCR charge density is mostly situated over the donor region while in LUMO is located over the $\pi$-linker and little bit over the donor. In the HOMO of HPPC7, charge density is mainly obtained over the donor and $\pi$-spacers fragment, while the LUMO charge density spread over the acceptor and $\pi$-spacer. In the designed 
compounds HPPC1-HPPC8, the large component of HOMOs was situated over donor portion while, small part on the $\pi$-spacers. Though, LUMOs are generally placed over the acceptor portion and partially over $\pi$-spacers. This shows that donor and acceptor units are linked with each other with the help of $\pi$-spacers, donor donates electron towards acceptor and $\pi$-spacers facilitate this transfer. This charge transfer phenomena reveals that all the studied compounds might be considered as impressive NLO constituents.

номо

HPPCR

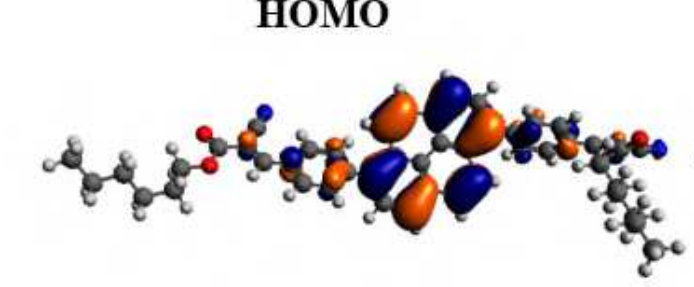

\section{LUMO}

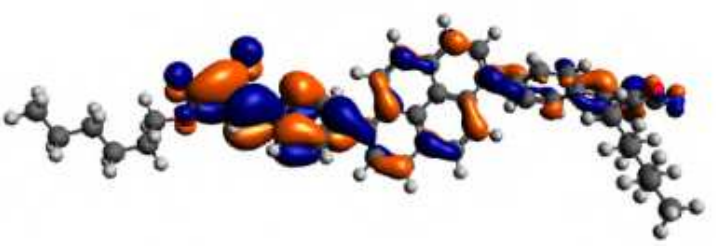

HPPC1

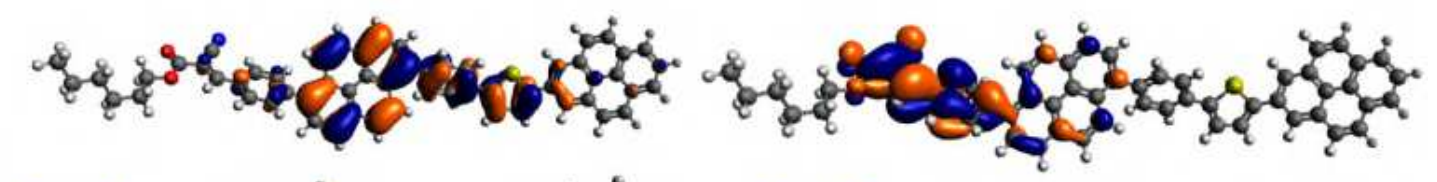

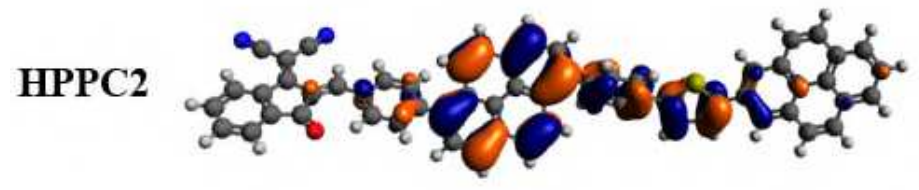

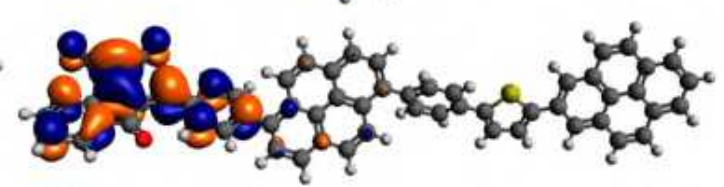

HРPC3
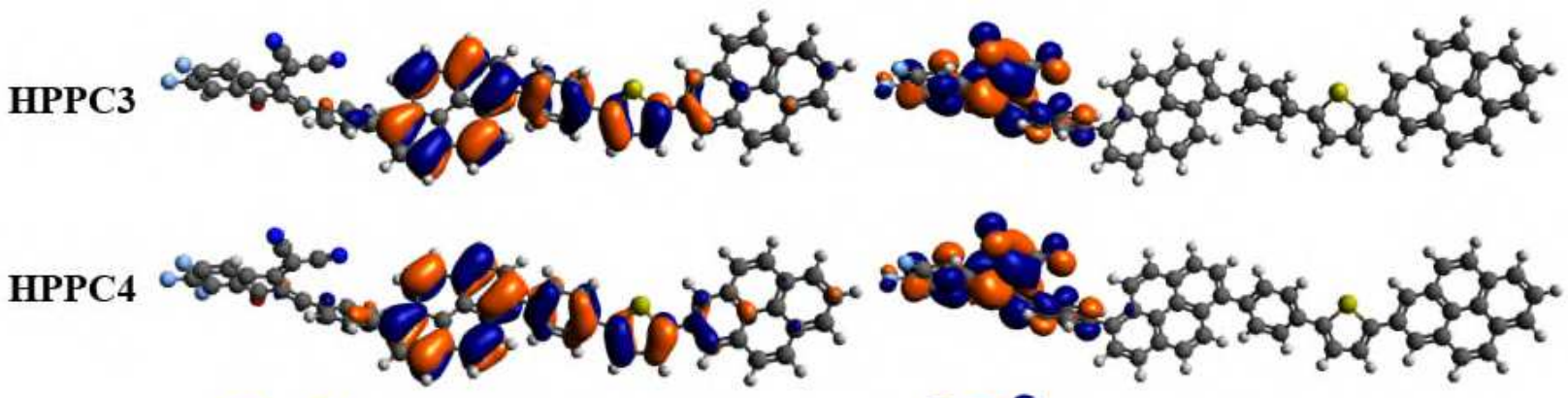

HPPC5
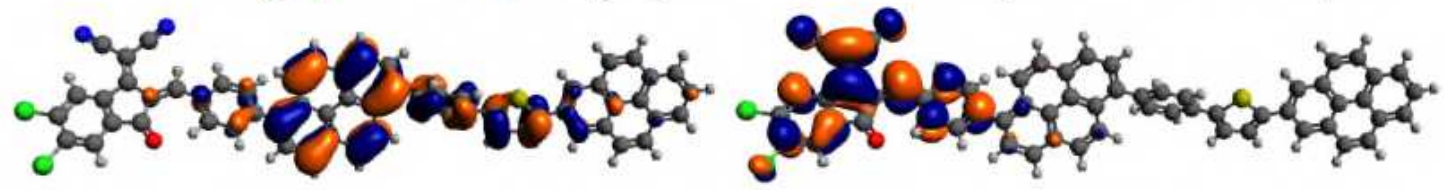

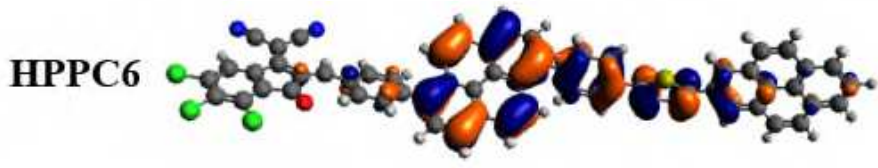
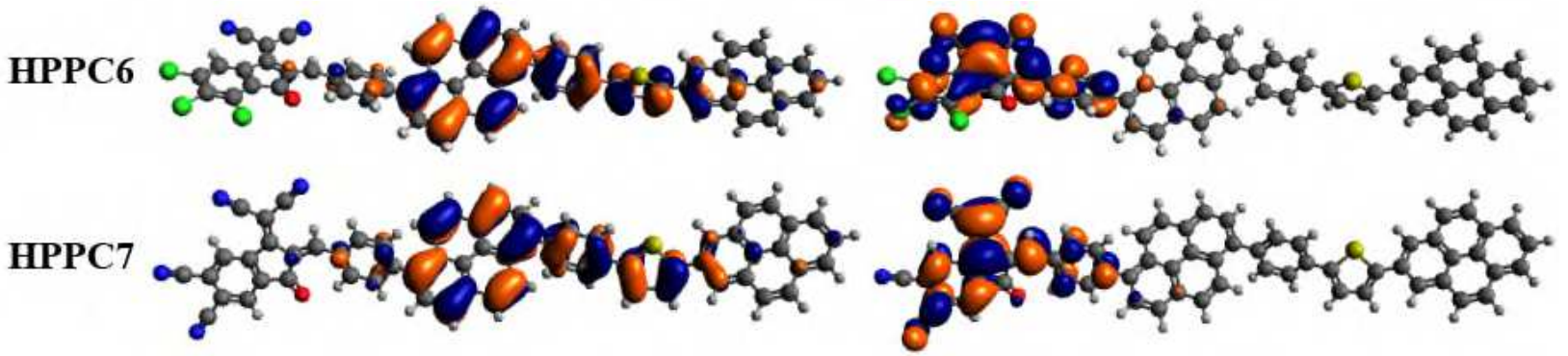

ures

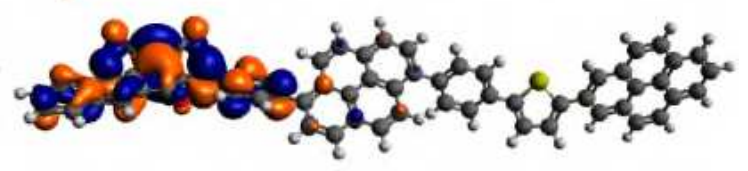


Figure 4: HOMOs and LUMOs of (HPPCR) and designed molecules (HPPC1-HPPC8).

\section{Global reactivity depicters}

The global reactivity depicters are very useful measuring tool for the stability and reactivity of designed molecules HPPC1-HPPC8 [47]. Global chemical reactivity descriptors, such as chemical potential $(\mu)$, global hardness $(\eta)$, global softness $(S)$, electronegativity $(\chi)$, and electrophilicity $(\omega)$, ionization potential $(I P)$ and electron affinity $(E A)$ have been determined by utilizing the HOMO LUMO band gap of designed compounds [48]. The ionization potential (I) denotes the energy required to remove an electron from the (HOMO) and the electron affinity (A) is the energy required to add an electron to the (LUMO) [47]. IP and EA are calculated by using the Koopman's equation [49].

$$
\begin{aligned}
& I P=-E_{\text {HOMO }} \\
& E A=-E_{\text {LUMO }}
\end{aligned}
$$

Ionization energy depicts the chemical reactivity of atoms and molecules. High ionization energy means high stability and chemical inertness and small ionization energy shows high reactivity of the atoms and molecules. Electron affinity refers to the capability of acceptor group to accept electron from a donor [50]. Table 2 reveals that $I P$ values of designed compound are smaller and $E A$ values are greater than the reference compound. These results showed that the HPPC1-HPPC8 might be soft, unstable and highly reactive compounds.

Table 2: Global reactivity parameters of all entitled compounds

\begin{tabular}{cccccccc}
\hline Compounds & $\boldsymbol{I P}$ & $\boldsymbol{E A}$ & $\boldsymbol{X}$ & $\boldsymbol{\eta}$ & $\boldsymbol{\mu}$ & $\boldsymbol{\omega}$ & $\sigma$ \\
\hline HPPCR & 0.211 & 0.092 & 0.152 & 0.059 & -0.152 & 0.195 & 8.438 \\
HPPC1 & 0.205 & 0.091 & 0.148 & 0.056 & -0.148 & 0.193 & 8.781 \\
HPPC2 & 0.205 & 0.113 & 0.159 & 0.046 & -0.159 & 0.275 & 10.836 \\
HPPC3 & 0.204 & 0.114 & 0.159 & 0.044 & -0.159 & 0.284 & 11.169 \\
HPPC4 & 0.204 & 0.117 & 0.160 & 0.043 & -0.160 & 0.297 & 11.497 \\
HPPC5 & 0.206 & 0.118 & 0.162 & 0.043 & -0.162 & 0.302 & 11.460 \\
HPPC6 & 0.205 & 0.121 & 0.163 & 0.042 & -0.163 & 0.318 & 11.896 \\
HPPC7 & 0.207 & 0.129 & 0.168 & 0.038 & -0.168 & 0.365 & 12.845 \\
HPPC8 & 0.205 & 0.115 & 0.160 & 0.044 & -0.160 & 0.286 & 11.136 \\
\hline
\end{tabular}

Units in Hartree $\left(\mathrm{E}_{\mathrm{h}}\right)$

Global hardness $(\boldsymbol{\eta})$ and global softness $(\boldsymbol{\sigma})$ are calculated by using following equations [51].

$$
\begin{gathered}
\eta=\frac{[I P-E A]}{2}=-\frac{\left[E_{\mathrm{LUMO}}-E_{\mathrm{HOMO}}\right]}{2} \\
\sigma=\frac{1}{2 \eta}
\end{gathered}
$$


From Table 2, values of softness and hardness reveals that all the investigated compounds HPPC1-HPPC8 can be considered to be the softest, most reactive, and most polarizable compounds, relative to the reference compound. Among all the designed compounds HPPC7 has lowest hardness value found to be 0.038 and highest softness value 12.845. Global hardness is decreased due to the substitution of $(-\mathrm{CN})$ group on end capped moiety. These $(-\mathrm{CN})$ groups enhance the electron withdrawing capacity of acceptor part and create a strong push pull mechanism within the compound, which in turn effects the stability of the compound and reduces its hardness.

The electronegativity of molecule is estimated by using the following equation [52].

$$
X=\frac{[I P+E A]}{2}=-\frac{\left[E_{\mathrm{LUMO}}+E_{\mathrm{HOMO}}\right]}{2}
$$

The electronegativity index signifies the attraction of electrons by the atom and functional group, which results in the transition of electrons from lower to higher electronegative part within the molecule [48]. The $\mu$ is the opposite of $\chi$.It is calculated by using the following equation [53].

$$
\mu=\frac{E_{\mathrm{HOMO}}+E_{\mathrm{LUMO}}}{2}
$$

Compounds having greater values of chemical potential are more reactive than those with small electronic chemical potentials.

The electrophilicity index is described as a structural depicter for the analysis of the chemical reactivity of molecules. It measures the tendency of the species to accept electrons. A good, more reactive, nucleophile has a lower value of $(\omega)$, in opposite a good electrophile has a high value of $(\omega)$. The electrophilicity index values are calculated by equation $[54,55]$.

$$
\omega=\frac{\mu^{2}}{2 \eta}
$$

Table 2 reveals all the investigated compound has higher electrophilicity index. Electrophilicity is considered to be good if the $\mu$ value is high and $\eta$ is low [56].

Consequently, HPPC1-HPPC8 may hold potential NLO findings. Furthermore, these findings of the designed molecules are linked with the energy gap values; the compounds with lower energy gap, showed smaller values of hardness and chemical potential with larger value of softness hence, more reactive and vice versa. So, all this information illustrates that the compounds may be naturally active and has noteworthy NLO properties. 


\section{UV-Vis analysis}

In order to comprehend the effect of different substituents on end capped acceptor moiety, observed spectral properties of designed compounds have been calculated by using TDDFT/M06/6-311G(d,p) for six excited states. These calculations were performed in DCM (dichloromethane) solvent for the approximation of six lowest singlet-singlet transitions. The computed absorption wavelengths $(\lambda)$ are function of electron availability, oscillator strengths $(f)$ and excitation energies $(E)$ were also calculated for the same solvent of compounds (HPPCR) and (HPPC1- HPPC8) are presented in Table S23, while major values are collected in Table 3.

Table 3: Transition energy $(E)$, maximum wavelengths $\left(\lambda_{\max }\right)$, oscillator strengths $\left(f_{o s}\right)$ and MO contributions

\begin{tabular}{cllll}
\hline Compounds & $\boldsymbol{\lambda}(\boldsymbol{n m})$ & $\mathbf{E}(\boldsymbol{e V})$ & $\boldsymbol{f}$ & MO contributions \\
\hline HPPCR & 471.764 & 2.628 & 1.085 & $\mathrm{H} \rightarrow \mathrm{L}(97 \%)$ \\
& $(405)^{\mathrm{b}} n m$ & & & \\
HPPC1 & 483.727 & 2.563 & 0.978 & $\mathrm{H} \rightarrow \mathrm{L}(92 \%), \mathrm{H}-2 \rightarrow \mathrm{L}(5 \%)$ \\
HPPC2 & 594.334 & 2.086 & 0.609 & $\mathrm{H} \rightarrow \mathrm{L}(92 \%), \mathrm{H}-2 \rightarrow \mathrm{L}(5 \%)$ \\
HPPC3 & 620.138 & 1.999 & 0.169 & $\mathrm{H} \rightarrow \mathrm{L}(92 \%), \mathrm{H}-2 \rightarrow \mathrm{L}(6 \%)$ \\
HPPC4 & 641.408 & 1.933 & 0.218 & $\mathrm{H} \rightarrow \mathrm{L}(92 \%), \mathrm{H}-2 \rightarrow \mathrm{L}(6 \%)$ \\
HPPC5 & 631.252 & 1.964 & 0.595 & $\mathrm{H} \rightarrow \mathrm{L}(92 \%), \mathrm{H}-2 \rightarrow \mathrm{L}(5 \%)$ \\
HPPC6 & 654.996 & 1.893 & 0.530 & $\mathrm{H} \rightarrow \mathrm{L}(92 \%), \mathrm{H}-2 \rightarrow \mathrm{L}(6 \%)$ \\
HPPC7 & 707.996 & 1.751 & 0.435 & $\mathrm{H} \rightarrow \mathrm{L}(92 \%), \mathrm{H}-2 \rightarrow \mathrm{L}(5 \%)$ \\
HPPC8 & 613.753 & 2.020 & 0.695 & $\mathrm{H} \rightarrow \mathrm{L}(92 \%), \mathrm{H}-2 \rightarrow \mathrm{L}(5 \%)$ \\
\hline
\end{tabular}

Values in parenthesis are experimental, [25] H = HOMO, H-2 = HOMO-2,L=LUMO,L-1 = LUMO-1

The maximum absorption wavelengths of all the designed molecules are observed in the range of 483-707 $\mathrm{nm}$. The computed results revealed that the strong electron withdrawing endcapped moieties have more extended conjugation, resulting in a greater red shift in the absorption spectra. The designed compounds have higher red shifted compared with reference HPPCR $\left(\lambda_{\max }=471 \mathrm{~nm}\right)$ as can be seen in Table 3. The decreasing order of absorption wavelengths for all investigated compounds along with the reference compound are found to be HPPC7> HPPC6> HPPC4> HPPC5> HPPC3> HPPC8> HPPC2> HPPC1> HPPCR.

The decreasing order of absorption wavelengths and energy gap is obtained to be the same for all the studied molecules. In fact, these compounds (HPPCR- HPPC7) have low energy gaps and demands small energy for the electronic transitions. Indeed, red shifted absorption wavelengths were found in HPPCR-HPPC7 due to low energy transitions. Interestingly, HPPCR-HPPC7 are also found in the range of environment friendly compounds owing to 
absorbance in the UV region $[57,58]$. It is expected that these compounds will immensely play a positive role in lowering the current global warming situation of the world. Moreover, the transition energy of all of the investigated compounds is obtained in the range of $2.628-1.751 \mathrm{eV}$. The lower transition energy is seen in HPPC7, which is due to the effect of strong end-capped acceptor moiety. The increasing order of transition energies are such as HPPC7 < HPPC6 < HPPC4 $<$ HPPC 5 <PPC3 < HPPC8 < HPPC2 <HPPC1 <HPPCR that is alike with oscillator strength. The molecular orbital calculations reveals that the visible absorption maxima of studied compounds relate to the electron transition from HOMO to LUMO. Major contribution in these molecular orbital transitions belongs to $\mathbf{H} \rightarrow \mathbf{L}$ (92\%). The molecular orbital transition reveals that maximum absorption spectra corelate with the transition from HUMO to LUMO. Furthermore, higher HOMO- LUMO compound shows deprived NLO response, while lowest HOMO-LUMO band gap compound HPPC7 establish maximum NLO response.

For the designing of NLO active materials with outstanding NLO response involves strong electronic coupling between donor and acceptor. Based on above discussion, (a) absorption spectra, (b) transition energy and (c) oscillator strength of designed compounds (HPPC1HPPC8) are much better than the reference compound HPPCR. These results proposed that, these type (A- $-\pi-\mathrm{D}-\pi-\mathrm{D})$ of designed compounds have marvelous NLO properties. The usage of the substituents has (-I) effect on acceptor moiety is a substantial approach. We anticipate that the effect of these non-fullerene acceptor units on NLO materials will be applied to design the NLO materials which performs a vital role in applied sciences.

\section{NBO analysis}

The natural bond orbital analysis was achieved by utilizing Gaussian NBO 6.0 program package [59] M06/6-31G(d,p) level of theory. NBO analysis allows us to evaluate: (i) the interaction between donor unit (D) and acceptor unit (A); (ii) the electronic excitation; and (iii) the electron delocalization [60]. The studied compounds are divided into D, $\pi$ (linker group) and A parts, although NBO analysis explains the transfer of charge density from donor(i) to acceptor (j) and $\pi$-linker acts as a conveyor for ICT charge transfer [61]. The stabilization energy $\mathrm{E}^{(2)}$ for delocalization can be attained by Eq12:

$$
E^{(2)}=q_{i} \frac{\left(F_{i, j}\right)^{2}}{\varepsilon_{j}-\varepsilon_{i}}
$$


Whereas, $q_{\mathrm{i}}$ is donor orbital occupancy, $\varepsilon_{i}$ and $\varepsilon_{\mathrm{j}}$ are off-diagonal and $F_{\mathrm{i} . \mathrm{j}}$ is diagonal NBO Fock matrix elements [62]. The NBO analysis offers useful understandings for analyzing the inter and intra-molecular hydrogen bonding, hyper conjugative interactions, charge transference between $\mathrm{D}$ and A units $[60,62]$. The designed compounds NBO analysis parameters are listed in the Table S11-S19 while major values are put in Table 4.

Table 4: Representative values of natural bond orbital analysis for HPPCR-HPPC8

\begin{tabular}{|c|c|c|c|c|c|c|c|}
\hline Compounds & Donor(i) & Type & Acceptor(j) & Type & $\mathbf{E}(2)^{\mathbf{a}}$ & $\mathbf{E}(\mathbf{J}) \mathbf{E}(\mathbf{i})^{\mathbf{b}}$ & $\mathbf{F}(\mathbf{i}, \mathbf{j})^{\mathrm{c}}$ \\
\hline \multirow{6}{*}{ HPPCR } & $\mathrm{C} 25-\mathrm{C} 26$ & $\pi$ & C28-C32 & $\pi^{*}$ & 27.13 & 0.29 & 0.079 \\
\hline & C77-O78 & $\pi$ & C77-O78 & $\pi^{*}$ & 0.71 & 0.43 & 0.017 \\
\hline & $\mathrm{C} 72-\mathrm{H} 73$ & $\sigma$ & C74-C77 & $\sigma^{*}$ & 7.41 & 0.96 & 0.076 \\
\hline & C90-C93 & $\sigma$ & C93-H97 & $\sigma^{*}$ & 0.50 & 1.04 & 0.020 \\
\hline & $\mathrm{O} 79$ & $\mathrm{LP}(2)$ & C77-O78 & $\pi^{*}$ & 52.51 & 0.36 & 0.124 \\
\hline & O79 & $\mathrm{LP}(2)$ & C63-C65 & $\pi^{*}$ & 0.54 & 0.38 & 0.013 \\
\hline \multirow{6}{*}{ HPPC1 } & C2-C3 & $\pi$ & C1-C6 & $\pi^{*}$ & 23.77 & 0.29 & 0.075 \\
\hline & C35-C37 & $\pi$ & C35-C37 & $\pi^{*}$ & 1.70 & 0.33 & 0.021 \\
\hline & C92-H103 & $\sigma$ & C86-C93 & $\sigma^{*}$ & 4.70 & 1.10 & 0.064 \\
\hline & C95-H96 & $\sigma$ & C83-C95 & $\sigma^{*}$ & 0.66 & 1.07 & 0.024 \\
\hline & $\mathrm{O} 42$ & $\mathrm{LP}(2)$ & $\mathrm{C} 40-\mathrm{O} 41$ & $\pi^{*}$ & 52.15 & 0.36 & 0.124 \\
\hline & $\mathrm{O} 42$ & $\mathrm{LP}(1)$ & C43-H46 & $\sigma^{*}$ & 0.93 & 1.01 & 0.027 \\
\hline \multirow{6}{*}{ HPPC2 } & C92-C94 & $\pi$ & C23-C73 & $\pi^{*}$ & 25.81 & 0.29 & 0.081 \\
\hline & C82-N83 & $\pi$ & C84-N85 & $\pi^{*}$ & 0.78 & 0.47 & 0.017 \\
\hline & C23-C79 & $\sigma$ & C73-C74 & $\sigma^{*}$ & 7.98 & 0.98 & 0.080 \\
\hline & C92-H96 & $\sigma$ & C23-C79 & $\sigma^{*}$ & 0.67 & 0.96 & 0.023 \\
\hline & S36 & $\mathrm{LP}(2)$ & C37-C39 & $\pi^{*}$ & 23.31 & 0.29 & 0.073 \\
\hline & O86 & $\mathrm{LP}(1)$ & C67-C74 & $\sigma^{*}$ & 1.35 & 1.19 & 0.036 \\
\hline \multirow{6}{*}{ HPPC3 } & C24-C25 & $\pi$ & C27-C30 & $\pi^{*}$ & 25.84 & 0.29 & 0.077 \\
\hline & C91-N92 & $\pi$ & C89-N90 & $\pi^{*}$ & 0.59 & 0.47 & 0.015 \\
\hline & C89-N90 & $\sigma$ & C88-C89 & $\sigma^{*}$ & 6.30 & 1.57 & 0.089 \\
\hline & C89-N90 & $\sigma$ & C84-C88 & $\sigma^{*}$ & 0.52 & 1.64 & 0.026 \\
\hline & $\mathrm{S} 46$ & $\mathrm{LP}(2)$ & C47-C49 & $\pi^{*}$ & 23.31 & 0.29 & 0.073 \\
\hline & O93 & $\mathrm{LP}(2)$ & C33-H87 & $\sigma^{*}$ & 0.59 & 0.70 & 0.019 \\
\hline \multirow{6}{*}{ HPPC4 } & C24-C26 & $\pi$ & C29-C30 & $\pi^{*}$ & 26.23 & 0.29 & 0.077 \\
\hline & C90-N91 & $\pi$ & C88-N89 & $\pi^{*}$ & 0.57 & 0.47 & 0.015 \\
\hline & C33-H86 & $\sigma$ & C83-C84 & $\sigma^{*}$ & 9.50 & 1.01 & 0.088 \\
\hline & C3-C7 & $\sigma$ & C7-CH18 & $\sigma^{*}$ & 0.80 & 1.13 & 0.027 \\
\hline & O92 & $\mathrm{LP}(2)$ & C76-C82 & $\sigma^{*}$ & 23.67 & 0.74 & 0.120 \\
\hline & F95 & $\mathrm{LP}(2)$ & C80-C81 & $\sigma^{*}$ & 0.51 & 0.98 & 0.020 \\
\hline \multirow{6}{*}{ HPPC5 } & C78-C80 & $\pi$ & C83-C85 & $\pi^{*}$ & 28.83 & 0.28 & 0.080 \\
\hline & C93-N94 & $\pi$ & C91-N92 & $\pi^{*}$ & 0.71 & 0.46 & 0.016 \\
\hline & C34-H37 & $\sigma$ & C33-S35 & $\sigma^{*}$ & 5.26 & 0.75 & 0.056 \\
\hline & C3-C6 & $\sigma$ & C6-H17 & $\sigma^{*}$ & 0.80 & 1.13 & 0.027 \\
\hline & S35 & $\mathrm{LP}(2)$ & C33-C34 & $\pi^{*}$ & 23.32 & 0.29 & 0.073 \\
\hline & $\mathrm{Cl} 76$ & $\operatorname{LP}(2)$ & C65-C70 & $\sigma^{*}$ & 0.55 & 0.91 & 0.020 \\
\hline
\end{tabular}




\begin{tabular}{llllllll}
\hline \multirow{5}{*}{ HPPC6 } & C78-C80 & $\pi$ & C83-C85 & $\pi^{*}$ & 29.12 & 0.28 & 0.081 \\
& C91-N92 & $\pi$ & C93-N94 & $\pi^{*}$ & 0.78 & 0.46 & 0.017 \\
& C93-N94 & $\sigma$ & C89-C93 & $\sigma^{*}$ & 6.27 & 1.57 & 0.089 \\
& C3-C6 & $\sigma$ & C3-H17 & $\sigma^{*}$ & 0.80 & 1.13 & 0.027 \\
& O90 & LP(2) & C66-C73 & $\sigma^{*}$ & 23.57 & 0.74 & 0.119 \\
& C176 & LP(2) & C65-C70 & $\sigma^{*}$ & 0.57 & 0.91 & 0.020 \\
\hline \multirow{5}{*}{ HPPC7 } & C25-C27 & $\pi$ & C25-C28 & $\pi^{*}$ & 29.59 & 0.28 & 0.081 \\
& C93-N94 & $\pi$ & C91-N92 & $\pi^{*}$ & 0.73 & 0.46 & 0.016 \\
& C35-H88 & $\sigma$ & C85-C86 & $\sigma^{*}$ & 7.86 & 0.98 & 0.079 \\
& C5-C16 & $\sigma$ & C16-H17 & $\sigma^{*}$ & 0.93 & 1.13 & 0.029 \\
& S48 & LP(2) & C46-C47 & $\pi^{*}$ & 23.24 & 0.29 & 0.073 \\
& O89 & LP(1) & C79-C86 & $\sigma^{*}$ & 1.39 & 1.17 & 0.036 \\
\hline \multirow{5}{*}{ HPPC8 } & C24-C26 & $\pi$ & C29-C30 & $\pi^{*}$ & 28.45 & 0.28 & 0.080 \\
& C90-N91 & $\pi$ & C88-N89 & $\pi^{*}$ & 0.73 & 0.46 & 0.016 \\
& C47-H50 & $\sigma$ & S46-C49 & $\sigma^{*}$ & 5.28 & 0.75 & 0.056 \\
& C3-C7 & $\sigma$ & C7-H18 & $\sigma^{*}$ & 0.80 & 1.13 & 0.027 \\
& S46 & LP(2) & C47-C49 & $\pi^{*}$ & 23.26 & 0.29 & 0.073 \\
& O86 & LP(1) & C77-C83 & $\sigma^{*}$ & 1.44 & 1.19 & 0.037 \\
\hline
\end{tabular}

${ }^{\mathrm{a}} \mathrm{E}^{(2)}$ means energy of hyper conjugative interaction (stabilization energy in $\mathrm{kcal} / \mathrm{mol}$ ). ${ }^{\mathrm{b}}$ Energy difference between donor and acceptor $\mathrm{i}$ and $\mathrm{j} \mathrm{NBO}$ orbitals $\mathrm{s} F(\mathrm{i} ; \mathrm{j})$ is the Fock matrix element between $\mathrm{i}$ and $\mathrm{j}$ NBO orbitals.

Typically, remarkable four types of molecular transitions were noticed; $\sigma \rightarrow \sigma^{*}, \pi \rightarrow \pi^{*}$, $\mathrm{LP} \rightarrow \sigma^{*}$ and $\mathrm{LP} \rightarrow \pi^{*}$. Transitions $\pi \rightarrow \pi^{*}$ was considered most significant, $\sigma \rightarrow \sigma^{*}$ was considered slightest and $\mathrm{LP} \rightarrow \sigma^{*} / \mathrm{LP} \rightarrow \pi^{*}$ were considered a little dominating transition. The extended conjugation and charge transfer in the designed molecules can be explained with $\pi \rightarrow$ $\pi^{*}$ transitions. The most noteworthy electronic interactions in terms of $\pi \rightarrow \pi^{*}$ such as $\pi\left(\mathrm{C}_{25}-\right.$ $\left.\mathrm{C}_{26}\right) \rightarrow \pi^{*}\left(\mathrm{C}_{28}-\mathrm{C}_{32}\right), \pi(\mathrm{C} 2-\mathrm{C} 3) \rightarrow \pi^{*}(\mathrm{C} 1-\mathrm{C} 6), \pi(\mathrm{C} 92-\mathrm{C} 94) \rightarrow \pi^{*}(\mathrm{C} 23-\mathrm{C} 73), \pi(\mathrm{C} 24-\mathrm{C} 25) \rightarrow \pi^{*}($ $\mathrm{C} 27-\mathrm{C} 30), \pi(\mathrm{C} 24-\mathrm{C} 26) \rightarrow \pi^{*}(\mathrm{C} 29-\mathrm{C} 30), \pi(\mathrm{C} 78-\mathrm{C} 80) \rightarrow \pi^{*}(\mathrm{C} 83-\mathrm{C} 85), \pi(\mathrm{C} 78-\mathrm{C} 80) \rightarrow \pi^{*}(\mathrm{C} 83-$ $\mathrm{C} 85), \pi(\mathrm{C} 25-\mathrm{C} 27) \rightarrow \pi^{*}(\mathrm{C} 25-\mathrm{C} 28), \pi(\mathrm{C} 24-\mathrm{C} 26) \rightarrow \pi^{*}(\mathrm{C} 29-\mathrm{C} 30)$ were obtained with stabilization energies $27.13,23.77,25.81,25.84,26.23,28.83,29.12,29.59,28.45 \mathrm{kcal} / \mathrm{mol}$ in HPPCR and HPPC1-HPPC8, respectively. All these excitations were found to be highest among all the transitions as occurred in HPPCR and its derivatives. However, transitions with lowest stabilization energies: $\pi(\mathrm{C} 77-\mathrm{O} 78) \rightarrow \pi^{*}(\mathrm{C} 77-\mathrm{O} 78), \pi(\mathrm{C} 35-\mathrm{C} 37) \rightarrow \pi^{*}(\mathrm{C} 35-\mathrm{C} 37), \pi(\mathrm{C} 82-\mathrm{N} 83) \rightarrow$ $\pi^{*}(\mathrm{C} 84-\mathrm{N} 85), \pi(\mathrm{C} 91-\mathrm{N} 92) \rightarrow \pi^{*}(\mathrm{C} 89-\mathrm{N} 90), \pi(\mathrm{C} 90-\mathrm{N} 91) \rightarrow \pi^{*}(\mathrm{C} 88-\mathrm{N} 89), \pi(\mathrm{C} 93-\mathrm{N} 94) \rightarrow \pi(\mathrm{C} 91-$ $\mathrm{N} 92), \pi(\mathrm{C} 91-\mathrm{N} 92) \rightarrow \pi^{*}(\mathrm{C} 93-\mathrm{N} 94), \pi(\mathrm{C} 93-\mathrm{N} 94) \rightarrow \pi^{*}(\mathrm{C} 91-\mathrm{N} 92), \pi(\mathrm{C} 90-\mathrm{N} 91) \rightarrow \pi^{*}(\mathrm{C} 88-\mathrm{N} 89)$ with $0.71,1.70,0.78,0.59,0.57,0.71,0.78,0.73$, and $0.73 \mathrm{kcal} / \mathrm{mol}$ stabilization energies were observed in HPPCR and HPPC1-HPPC8 respectively.

In the same way, $\sigma \rightarrow \sigma^{*}$ transitions were examined: $\sigma(\mathrm{C} 72-\mathrm{H} 73) \rightarrow \sigma^{*}(\mathrm{C} 74-\mathrm{C} 77), \sigma(\mathrm{C} 92-$ $\mathrm{H} 103) \rightarrow \quad \sigma^{*}(\mathrm{C} 86-\mathrm{C} 93), \quad \sigma(\mathrm{C} 23-\mathrm{C} 79) \rightarrow \quad \sigma^{*}(\mathrm{C} 73-\mathrm{C} 74), \sigma(\mathrm{C} 89-\mathrm{N} 90) \rightarrow \quad \sigma^{*}(\mathrm{C} 88-\mathrm{C} 89), \quad \sigma(\mathrm{C} 33-$ 
$\mathrm{H} 86) \rightarrow \sigma^{*}(\mathrm{C} 83-\mathrm{C} 84), \quad \sigma(\mathrm{C} 34-\mathrm{H} 37) \rightarrow \quad \sigma^{*}(\mathrm{C} 33-\mathrm{S} 35), \quad \sigma(\mathrm{C} 93-\mathrm{N} 94) \rightarrow \sigma^{*}(\mathrm{C} 89-\mathrm{C} 93), \quad \sigma(\mathrm{C} 35-$ $\mathrm{H} 88) \rightarrow \sigma^{*}(\mathrm{C} 85-\mathrm{C} 86)$, and $\sigma(\mathrm{C} 47-\mathrm{H} 50) \rightarrow \sigma^{*}(\mathrm{~S} 46-\mathrm{C} 49)$ with $7.41,4.70,7.98,6.30,9.50,5.26$, 6.27, 7.86, and $5.28 \mathrm{kcal} / \mathrm{mol}$ stabilization energies in HPPCR and HPPC1-HPPC8, respectively. Above values were the largest values among all the $\sigma \rightarrow \sigma^{*}$ excitations of HPPCR and HPPC1-HPPC8. While $\sigma(\mathrm{C} 90-\mathrm{C} 93) \rightarrow \sigma^{*}(\mathrm{C} 93-\mathrm{H} 97), \sigma(\mathrm{C} 95-\mathrm{H} 96) \rightarrow \sigma^{*}(\mathrm{C} 83-\mathrm{C} 95), \sigma(\mathrm{C} 92-$ $\mathrm{H} 96) \rightarrow \sigma^{*}(\mathrm{C} 23-\mathrm{C} 79), \sigma(\mathrm{C} 89-\mathrm{N} 90) \rightarrow \sigma^{*}(\mathrm{C} 84-\mathrm{C} 88), \sigma(\mathrm{C} 3-\mathrm{C} 7) \rightarrow \sigma^{*}(\mathrm{C} 7-\mathrm{CH} 18), \sigma(\mathrm{C} 3-\mathrm{C} 6) \rightarrow$ $\sigma^{*}(\mathrm{C} 6-\mathrm{H} 17), \sigma(\mathrm{C} 3-\mathrm{C} 6) \rightarrow \sigma^{*}(\mathrm{C} 3-\mathrm{H} 17), \sigma(\mathrm{C} 5-\mathrm{C} 16) \rightarrow \sigma^{*}(\mathrm{C} 16-\mathrm{H} 17)$, and $\sigma(\mathrm{C} 3-\mathrm{C} 7) \rightarrow \sigma^{*}(\mathrm{C} 7-\mathrm{H} 18)$ with $0.50,0.66,0.67,0.52,0.80,0.80,0.80,0.93$, and $0.80 \mathrm{kcal} / \mathrm{mol}$ stabilization energies were found least values among all $\sigma \rightarrow \sigma^{*}$ transitions in HPPCR and HPPC1-HPPC8, respectively.

Furthermore, some important interactions were observed like LP2 (O79) $\rightarrow \pi^{*}\left(\mathrm{C}_{77}-\mathrm{O} 78\right)$, $\mathrm{LP} 2(\mathrm{O} 42) \rightarrow \quad \pi^{*}(\mathrm{C} 40-\mathrm{O} 41), \quad \mathrm{LP} 2(\mathrm{~S} 36) \rightarrow \quad \pi^{*}(\mathrm{C} 37-\mathrm{C} 39), \quad$ LP2(S46) $\rightarrow \pi^{*}(\mathrm{C} 47-\mathrm{C} 49), \quad$ LP2 $(\mathrm{O} 92) \rightarrow \sigma^{*}(\mathrm{C} 76-\mathrm{C} 82), \mathrm{LP} 2(\mathrm{~S} 35) \rightarrow \pi^{*}(\mathrm{C} 33-\mathrm{C} 34), \mathrm{LP} 2(\mathrm{O} 90) \rightarrow \sigma^{*}(\mathrm{C} 66-\mathrm{C} 73) \quad \mathrm{LP} 2(\mathrm{~S} 48) \rightarrow$ $\pi^{*}(\mathrm{C} 46-\mathrm{C} 47)$, and LP2(S46) $\rightarrow \pi^{*}(\mathrm{C} 47-\mathrm{C} 49)$ contained $52.51,52.15,23.31,23.31,23.67,23.32$ ,23.57, 23.24 and $23.26 \mathrm{kcal} / \mathrm{mol}$ in HPPCR and HPPC1-HPPC8, respectively. These LP $\rightarrow \pi^{*}$ were collected as highest values among all of LP $\rightarrow \pi^{*}$ transitions. On the other hand, LP2 $(\mathrm{O} 79) \rightarrow \quad \sigma^{*} \quad(\mathrm{C} 63-\mathrm{C} 65), \quad \mathrm{LP} 1(\mathrm{O} 42) \rightarrow \quad \sigma^{*} \quad(\mathrm{C} 43-\mathrm{H} 46), \quad \mathrm{LP} 1(\mathrm{O} 86) \rightarrow \sigma^{*}(\mathrm{C} 67-\mathrm{C} 74)$, $\mathrm{LP} 2(\mathrm{O} 93) \rightarrow \sigma^{*}(\mathrm{C} 33-\mathrm{H} 87), \quad \mathrm{LP} 2(\mathrm{~F} 95) \rightarrow \sigma^{*}(\mathrm{C} 80-\mathrm{C} 81), \quad \mathrm{LP} 2(\mathrm{Cl} 76) \rightarrow \quad \sigma^{*}(\mathrm{C} 65-\mathrm{C} 70)$, $\mathrm{LP} 2(\mathrm{Cl} 76) \rightarrow \sigma^{*}(\mathrm{C} 65-\mathrm{C} 70), \mathrm{LP} 1(\mathrm{O} 89) \rightarrow \sigma^{*}(\mathrm{C} 79-\mathrm{C} 86)$, and $\quad \mathrm{LP} 1(\mathrm{O} 86) \rightarrow \sigma^{*}(\mathrm{C} 77-\mathrm{C} 83)$ contained $0.54,0.93,1.35,0.59,0.51,0.55,0.57,1.39$ and $1.44 \mathrm{kcal} / \mathrm{mol}$ in HPPCR and HPPC1-HPPC8, respectively. These $\mathrm{LP} \rightarrow \pi^{*}$ were collected as least values in terms of magnitudes among all of $\mathrm{LP} \rightarrow \pi^{*}$ transitions. The NBO results show the conjugation, hyperconjugation and intramolecular charge transfer (ICT) phenomena is found in our HPPCR and HPPC1-HPPC8. Moreover, these NBO results also support NLO responses of the studied molecules [63].

\section{Non-Linear Optics}

Nonlinear materials along their investigations are gathering significance in various scientific aspects because of their huge role in an extensive diversity of applications ranging from lasers to signal processing and optical sensing devices [64-66]. We have computed the dipole moment ( $\mu_{\text {total }}$, linear polarizability $(\alpha)$, first $(\beta)$, and second $(\gamma)$ hyperpolarizabilities of the designed compounds HPPC1-HPPC8 at M06/6-311g(d, p). These are the important parameters which indicate their usefulness as NLO active materials [67]. They are theoretically 
calculated by employing $\mathrm{x}, \mathrm{y}, \mathrm{z}$ tensors presented in Eq. (1-4). The strength of the optical response correlate directly with the electronic properties and these properties are in good agreement. The computed $\mu, \alpha, \beta$ and $\gamma$ values exhibited in Table 5, and their contributing tensors are mentioned in Tables S20-S22.

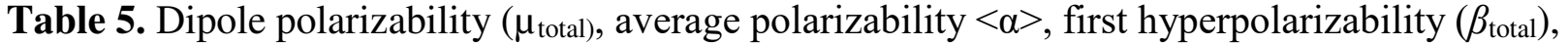
and second hyperpolarizability $\langle\gamma\rangle$ of the studied compounds.

\begin{tabular}{lllll} 
Compounds & $\mu_{\text {total }}$ & $\langle\alpha\rangle$ & $\beta_{\text {total }}$ & $\langle\gamma\rangle \times 10^{7}$ \\
\hline HPPCR & 4.275 & 854.100 & 8501.7 & 0.555 \\
HPPC1 & 3.537 & 1089.330 & 37856.5 & 0.796 \\
HPPC2 & 2.687 & 1230.656 & 98320.3 & 1.955 \\
HPPC3 & 1.955 & 1161.027 & 38409.2 & 1.050 \\
HPPC4 & 2.006 & 1177.857 & 56003.9 & 1.364 \\
HPPC5 & 3.308 & 1296.011 & 127270.7 & 2.656 \\
HPPC6 & 2.942 & 1323.223 & 141902.9 & 3.033 \\
HPPC7 & 5.666 & 1331.191 & 200112.2 & 4.131 \\
HPPC8 & 2.842 & 1344.056 & 120047.7 & 2.653 \\
\hline
\end{tabular}

At molecular level, the dipole moment is an important parameter to determine the role of acceptor group and also used to quantify the response of an isolate compound in an applied electric field. The non-zero values of $\mu_{\text {total }}$ depicts the dipolar character of investigated compounds. There are three tensors of the dipole polarizability $(\mu)$ along the directions of $\mathrm{x}, \mathrm{y}$ and $\mathrm{z}$ axes. Table S20 reveals that $\mu_{\mathrm{y}}$ tensor of HPPCR, HPPC1-HPPC4 and HPPC8 consisting of most significant values. Moreover, HPPC5-HPPC7 consist of most significant values of $\mu_{\mathrm{x}}$. However, all HPPCR and HPPC1-HPPC8 molecules comprise of lowest value of $\mu_{\mathrm{z}}$. The dipole moment values of reference compound HPPCR along with the designed compounds HPPC1-HPPC8 in DCM solvent are found to be 4.275, 3.537, 2.687, 1.955, 2.006, 3.308, 2.942, 5.666 and 2.842 respectively.

Among HPPCR and HPPC1-HPPC8, HPPC7 has the highest value of $\mu_{\text {total }}$ is owing to the strong electron withdrawing effect of non-fullerene acceptor moiety. The computed dipole moment of all the designed compounds is larger as compared to HPPCR. The total dipole polarizability $(\boldsymbol{\mu})$ decreasing trend is found to be HPPC7> HPPCR> HPPC1> HPPC5> HPPC6> HPPC8> HPPC2> HPPC4> HPPC3, disclosed that all the designed compounds have significantly polar behavior. Hence, it is clear from the result that larger the values of dipole moment greater will be the ICT charge transfer and therefore greater electron mobility. Therefore, all our studied compounds are enriched in this aspect. Linear polarizability $(\alpha)$ has three tensors in the directions of $\mathrm{x}, \mathrm{y}$ and $\mathrm{z}$ axes. Table S20 reveals that $\alpha_{\mathrm{xx}}$ tensor of HPPCR, HPPC1-HPPC8 consist of most significant values. However, all HPPC1-HPPC8 molecules 
comprise of the lowest values of $\mu_{\mathrm{zz}}$ and HPPCR comprise of lowest values of $\mu_{\mathrm{yy}}$. The HPPCR $(\alpha)$ value was found to be 854.10(a.u) and designed HPPC1-HPPC8 compounds have values: 1089.330, 1230.656, 1161.027, 1177.857, 1296.011, 1323.223, 1331.191 and 1344.056a.u respectively. Linear polarizability trend was found to be HPPC8> HPPC7> HPPC6> HPPC5> HPPC2> HPPC4> HPPC3> HPPC1> HPPCR, revealed that all the designed compounds are more effective than the reference compound in terms of linear polarizability $(\alpha)$ responses.

The first hyperpolarizability is represented by a third rank tensor by characterizing the response of a system in an applied electric field [68]. Table S21 reveals that $\beta_{\mathrm{xxx}}$ tensor of HPPCR, HPPC1-HPPC8 consist of most significant values. Moreover, $\beta_{\mathrm{xxy}}$ also consist of significant values but less than $\beta_{\mathrm{xxx}}$. Therefore, main charge transfer in HPPCR, HPPC1HPPC8 are found in the direction of x-axis. Moreover, Table S21, all HPPC1-HPPC8 have significant values of second order polarizability tensors as compared to HPPCR. This data suggests that the studied systems (HPPC1-HPPC8), other than reference system (HPPCR), have strong second-order polarizability responses. Second-order polarizabilities $(\beta)$ exemplify the NLO responses in which HPPC7 exhibited highest 200112.2a.u and found least as 8501a.u by HPPCR. In HPPC7, four CN groups on the acceptor moiety enhance the charge transfer ability by lowering the transition energy which in turn increases the NLO response of first hyperpolarizability. Large values in $\beta_{\text {total }}$ are observed to be 37856.5, 98320.3, 38409.2, 56003.9, 127270.7, 141902.9, 200112.2, and 120047.7a.u for HPPC1- HPPC8, respectively as compared to HPPCR (8501.7). This data proved that the efficacy of strong acceptor moieties was noticed remarkably in HPPC1- HPPC8. Moreover, linear polarizability and $\beta$ values of our designed compounds are found remarkably greater than urea, as a reference compound for the estimation of NLO response [69]. The $\beta$ values of HPPCR, HPPC1, HPPC2, HPPC3, HPPC4, HPPC5, HPPC6, HPPC7 and HPPC8 were 197 times, 880 times, 2286 times, 893 times, 1302 times, 2959 times, 3300 times, 4653 and 2791 times greater than urea, respectively, indicating high performance NLO active compounds. Indeed, second order NLO response correlate with the ICT in which electrons migrated through $\pi$-bridge towards the $\mathrm{x}$-axis. However, higher delocalization of $\pi$-electrons decreased the band gaps, which in turn increased NLO response for the compounds [70]. The second hyperpolarizability $(\gamma)$ is often thought as two-photon absorption (TPA) phenomenon in NLO compounds. Table S22 reveals that $\gamma_{X}$ tensor of HPPCR, HPPC1HPPC8 consist of most significant values. However, $\gamma_{z}$ consist of lowest values for HPPCR, 
HPPC1-HPPC8. Therefore, main charge transfer in HPPCR-HPPC8 is found in the direction of X-axis. The amplitude of $\gamma_{X}$ tensor for HPPC2-HPPC8 has greater magnitude than HPPC1 and HPPCR. All the investigated molecules show high third-order NLO response as compared to HPPCR. Third-order polarizability values of reference and designed compounds are 0.555 , $0.796,1.955,1.050,1.364,2.656,3.033,4.131$ and 2.653 all values of $10^{7}$ a.u respectively. All designed compounds are in the following decreasing order: HPPC7> HPPC6> HPPC5> HPPC8 $>$ HPPC2 $>$ HPPC4> HPPC3 $>$ HPPC1 $>$ HPPCR.

Based on the above discussion, dipole moment, polarizability and hyperpolarizability of all the designed compounds are greater than the reference compound. This increase is owing to the incorporation of electron withdrawing groups on accepter unit. These electron loving groups attract the electrons from the donor unit through $\pi$-spacers by lowering the transition energy, band gap and enhance the NLO response. So, we conclude that the designed compounds show promising NLO properties which provides basis for future studies.

\section{Conclusion}

In this work, the structural tailoring with acceptor groups remarkably affects the NLO properties of compounds has been reported. A series of chromophores (HPPC1-HPPC8) with A $-\pi-\mathrm{D}-\pi-\mathrm{D}$ architecture have been designed via different end-capped acceptor moieties, where pyrene ring acts as a donor unit and phenyl as well as thienyl act as $\pi$-bridges. The computed data for excitation energies reveals that all the investigated compounds have extensive electron delocalization as compared to the HPPCR. The band gap is ascertained in the range of 3.225$2.118 \mathrm{eV}$ for HPPC1-HPPC8. This energy difference communicated a clue about the stability and chemical reactivity of designed molecules. NBO result illustrates the charge transfer phenomena anticipated in HPPC1-HPPC8. In dichloromethane (DCM), the maximum bathochromic shift is observed as 707.996n for HPPC7 and has the smallest band gap is established as $2.118 \mathrm{eV}$. Consequently, HPPC7 has a more promising NLO than HPPCR and HPPC1-HPPC8. The total dipole polarizability $(\mu)$ trend is found to be HPPC7> HPPCR> HPPC1> HPPC5> HPPC6> HPPC8> HPPC2> HPPC4> HPPC3, disclosed that all the designed compounds have significantly polar behavior. Trend of linear polarizability $(\alpha)$ and first hyperpolarizability $(\beta)$ was found to be HPPC8> HPPC7> HPPC6> HPPC5> HPPC2> HPPC4> HPPC3> HPPC1> HPPCR. All designed molecules are in the following decreasing order of second hyperpolarizability $(\gamma)$ : HPPC7> HPPC6> HPPC5> HPPC8> HPPC2> 
HPPC4> HPPC3> HPPC1> HPPCR. Overall, all investigated compounds (HPPC1-HPPC8) have shown marvelous NLO response as compared to HPPCR. These theoretical findings might attract contemporary researchers to use modern approaches for synthesis of such highperformance NLO compounds.

\section{Acknowledgement}

"This research work was funded by Institutional Fund Projects under grant no. (IFPIP:1229-1301442). Therefore, the authors gratefully acknowledge technical and financial support from the Ministry of Education and King Abdulaziz University, Jeddah, Saudi Arabia"

\section{References}

1. Thukral, K., et al., Analyses of significant features of L-Prolinium Picrate single crystal: An excellent material for non linear optical applications. Materials Chemistry and Physics, 2017. 194: p. 90-96.

2. Nagaraja, H., et al., Organic nonlinear optical crystals of benzoyl glycine. Journal of crystal growth, 1998. 193(4): p. 674-678.

3. Srinivasan, P., et al., 2, 4, 6-trinitrophenol (TNP): An organic material for nonlinear optical (NLO) applications. Journal of crystal growth, 2006. 289(2): p. 639-646.

4. Vijayan, N., et al., A comparative study on solution-and bridgman-grown single crystals of benzimidazole by high-resolution X-ray diffractometry, fourier transform infrared, microhardness, laser damage threshold, and second-harmonic generation measurements. Crystal growth \& design, 2006. 6(6): p. 1542-1546.

5. Scarola, M., et al., Optical limiting and switching of ultrashort pulses in nonlinear photonic bandgap material. Phys. Rev. Lett., 1994. 73: p. 1368-1371.

6. Kumar, M.S., et al., Structural characteristics and second harmonic generation in Lthreonine crystals. Journal of crystal Growth, 2006. 286(2): p. 451-456.

7. Zyss, J. and J.-F. Nicoud, Status and perspective for molecular nonlinear optics: from crystals to polymers and fundamentals to applications. Current Opinion in Solid State and Materials Science, 1996. 1(4): p. 533-546.

8. Prasad, P.N. and D.J. Williams, Introduction to nonlinear optical effects in molecules and polymers. Vol. 1. 1991: Wiley New York. 
9. Thanthiriwatte, K.S. and K.N. De Silva, Non-linear optical properties of novel fluorenyl derivatives-ab initio quantum chemical calculations. Journal of Molecular Structure: THEOCHEM, 2002. 617(1-3): p. 169-175.

10. Kobayashi, T., Nonlinear optics of organics and semiconductors: proceedings of the international symposium, Tokyo, Japan, July 25-26, 1988. Vol. 36. 1989: Springer Verlag.

11. Chemla, D.S., Nonlinear Optical Properties of Organic Molecules and Crystals V1. Vol. 1. 2012: Elsevier.

12. Hann, R. and D. Bloor, Organic materials for non-linear optics II. Vol. 2. 1991: CRC PressI Llc.

13. Eaton, D.F., Nonlinear optical materials. Science, 1991. 253(5017): p. 281-287.

14. Kanis, D.R., M.A. Ratner, and T.J. Marks, Design and construction of molecular assemblies with large second-order optical nonlinearities. Quantum chemical aspects. Chemical Reviews, 1994. 94(1): p. 195-242.

15. Khalid, M., et al., First principles study of electronic and nonlinear optical properties of $A-D-\pi-A$ and $D-A-D-\pi-A$ configured compounds containing novel quinoline-carbazole derivatives. RSC Advances, 2020. 10(37): p. 22273-22283.

16. Fuchs, B.A., C.K. Syn, and S.P. Velsko, Diamond turning of L-arginine phosphate, a new organic nonlinear crystal. Applied optics, 1989. 28(20): p. 4465-4472.

17. Kamanina, N.V., et al., Polyimide-fullerene nanostructured materials for nonlinear optics and solar energy applications. Journal of Materials Science: Materials in Electronics, 2012. 23(8): p. 1538-1542.

18. Li, M., et al., Solution-processed organic tandem solar cells with power conversion efficiencies $>12 \%$. Nature Photonics, 2017. 11(2): p. 85-90.

19. Speller, E.M., et al., From fullerene acceptors to non-fullerene acceptors: prospects and challenges in the stability of organic solar cells. Journal of Materials Chemistry A, 2019. 7(41): p. 23361-23377.

20. McAfee, S.M., et al., Key components to the recent performance increases of solution processed non-fullerene small molecule acceptors. Journal of Materials Chemistry A, 2015. 3(32): p. 16393-16408. 
21. Nielsen, C.B., et al., Non-fullerene electron acceptors for use in organic solar cells. Accounts of chemical research, 2015. 48(11): p. 2803-2812.

22. Li, S., et al., Efficient organic solar cells with non-fullerene acceptors. Small, 2017. 13(37): p. 1701120.

23. Hou, J., et al., Organic solar cells based on non-fullerene acceptors. Nature materials, 2018. 17(2): p. 119-128.

24. Yang, Y., et al., Side-chain isomerization on an n-type organic semiconductor ITIC acceptor makes $11.77 \%$ high efficiency polymer solar cells. Journal of the American Chemical Society, 2016. 138(45): p. 15011-15018.

25. Nan, M.I., et al., Mono-and di-substituted pyrene-based donor- $\pi$-acceptor systems with phenyl and thienyl $\pi$-conjugating bridges. Dyes and Pigments, 2020. 181: p. 108527.

26. Wielopolski, M., et al., Position-dependent extension of $\pi$-conjugation in $D-\pi$-A dye sensitizers and the impact on the charge-transfer properties. The Journal of Physical Chemistry C, 2013. 117(27): p. 13805-13815.

27. Katono, M., et al., Effect of extended $\pi$-conjugation of the donor structure of organic $D$ $A-\pi-A$ dyes on the photovoltaic performance of dye-sensitized solar cells. The Journal of Physical Chemistry C, 2014. 118(30): p. 16486-16493.

28. Panneerselvam, M., et al., The role of $\pi$-linkers in tuning the optoelectronic properties of triphenylamine derivatives for solar cell applications-A DFT/TDDFT study. Physical Chemistry Chemical Physics, 2017. 19(8): p. 6153-6163.

29. Namuangruk, S., et al., $D-D-\pi-A$-Type organic dyes for dye-sensitized solar cells with a potential for direct electron injection and a high extinction coefficient: synthesis, characterization, and theoretical investigation. The Journal of Physical Chemistry C, 2012. 116(49): p. 25653-25663.

30. Rotzler, J., et al., Variation of the Backbone Conjugation in NLO Model Compounds: Torsion-Angle-Restricted, Biphenyl-Based Push-Pull-Systems, 2010, Wiley Online Library.

31. Cardelino, B.H., C.E. Moore, and R.E. Stickel, Static second-order polarizability calculations for large molecular systems. The Journal of Physical Chemistry, 1991. 95(22): p. 8645-8652.

32. Frisch, M., et al., Gaussian, 16 version. Gaussian Inc: Wallingford, CT, USA, 2016. 
33. Dennington, R., T.A. Keith, and J.M. Millam, GaussView, version 6.0. 16. Semichem Inc. Shawnee Mission KS, 2016.

34. Barone, V. and M. Cossi, Quantum calculation of molecular energies and energy gradients in solution by a conductor solvent model. The Journal of Physical Chemistry A, 1998. 102(11): p. 1995-2001.

35. Hanwell, M.D., et al., Avogadro: an advanced semantic chemical editor, visualization, and analysis platform. Journal of cheminformatics, 2012. 4(1): p. 1-17.

36. Zhurko, G., Chemcraft: http://www. chemcraftprog. com. Received: October, 2014. 22.

37. Valverde, C., et al., Third-order nonlinear optical properties of a carboxylic acid derivative. Acta Chimica Slovenica, 2018. 65(3): p. 739-749.

38. Risser, S.M., D.N. Beratan, and S.R. Marder, Structure-function relationships for. beta., the first molecular hyperpolarizability. Journal of the American Chemical Society, 1993. 115(17): p. 7719-7728.

39. Solomon, R.V., et al., Tuning nonlinear optical and optoelectronic properties of vinyl coupled triazene chromophores: a density functional theory and time-dependent density functional theory investigation. J Phys Chem A, 2012. 116(18): p. 4667-77.

40. Albayrak, Ç. and R. Frank, Spectroscopic, molecular structure characterizations and quantum chemical computational studies of (E)-5-(diethylamino)-2-[(2fluorophenylimino)methyl]phenol. Journal of Molecular Structure, 2010. 984(1-3): p. 214-220.

41. Almutairi, M.S., et al., Spectroscopic (FT-IR, FT-Raman, UV, $1 H$ and 13C NMR) profiling and computational studies on methyl 5-methoxy-1H-indole-2-carboxylate: A potential precursor to biologically active molecules. Journal of Molecular Structure, 2017. 1133: p. 199-210.

42. Sajan, D., et al., Vibrational spectra and first-order molecular hyperpolarizabilities of phydroxybenzaldehyde dimer. Journal of Molecular Structure, 2010. 983(1-3): p. 12-21.

43. Maidur, S.R., et al., Experimental and computational studies on second-and third-order nonlinear optical properties of a novel D- $\pi$-A type chalcone derivative: 3-(4methoxyphenyl)-1-(4-nitrophenyl) prop-2-en-1-one. Optics \& Laser Technology, 2017. 97: p. 219-228. 
44. A, A.P., K. S, and M. S.P, Supramolecular architecture of third-order nonlinear optical ammonium picrate: Crystal growth and DFT approach. Optik, 2016. 127(15): p. 61346149.

45. Katariya, S., et al., Triphenylamine-Based Fluorescent Styryl Dyes: DFT, TD-DFT and Non-Linear Optical Property Study. J Fluoresc, 2017. 27(3): p. 993-1007.

46. Fukui, K., Role of frontier orbitals in chemical reactions. science, 1982. 218(4574): p. 747-754.

47. Dheivamalar, S., et al., Adsorption of alanine with heteroatom substituted fullerene for solar cell application: A DFT study. Spectrochimica Acta Part A: Molecular and Biomolecular Spectroscopy, 2018. 202: p. 333-345.

48. Yousif, A.A. and G.F. Fadhil, DFT of para methoxy dichlorochalcone isomers. Investigation of structure, conformation, FMO, charge, and NLO properties. Chemical Data Collections, 2021. 31: p. 100618.

49. Koopmans, T., Ordering of wave functions and eigenenergies to the individual electrons of an atom. Physica, 1933. 1: p. 104-113.

50. Meenakshi, R., Spectral investigations, DFT based global reactivity descriptors, Inhibition efficiency and analysis of 5-chloro-2-nitroanisole as $\pi$-spacer with donoracceptor variations effect for DSSCs performance. Journal of Molecular Structure, 2017. 1127: p. 694-707.

51. Pearson, R.G., Chemical hardness. 1997: Wiley-VCH.

52. Pritchard, H. and H. Skinner, The concept of electronegativity. Chemical Reviews, 1955. 55(4): p. 745-786.

53. Parr, R.G., et al., Electronegativity: the density functional viewpoint. The Journal of Chemical Physics, 1978. 68(8): p. 3801-3807.

54. Maynard, A., et al., Reactivity of the HIV-1 nucleocapsid protein $p 7$ zinc finger domains from the perspective of density-functional theory. Proceedings of the National Academy of Sciences, 1998. 95(20): p. 11578-11583.

55. Parr, R.G., L.v. Szentpály, and S. Liu, Electrophilicity index. Journal of the American Chemical Society, 1999. 121(9): p. 1922-1924.

56. Kumar, A., et al., Molecular structure, nonlinear optical studies and spectroscopic analysis of chalcone derivative (2E)-3-[4-(methylsulfanyl) phenyl]-1-(3-bromophenyl) 
prop-2-en-1-one by DFT calculations. Journal of Molecular Structure, 2017. 1150: p. 166-178.

57. Tomkinson, J., et al., The vibrational spectroscopy of indigo: A reassessment. Vibrational Spectroscopy, 2009. 50(2): p. 268-276.

58. Bechtold, T. and R. Mussak, Handbook of natural colorants. 2009: John Wiley \& Sons.

59. Glendening, E.D., C.R. Landis, and F. Weinhold, NBO 6.0: Natural bond orbital analysis program. Journal of computational chemistry, 2013. 34(16): p. 1429-1437.

60. Ans, M., et al., Designing Three-dimensional (3D) Non-Fullerene Small Molecule Acceptors with Efficient Photovoltaic Parameters. ChemistrySelect, 2018. 3(45): p. 12797-12804.

61. Bribes, J.L., M. El Boukari, and J. Maillols, Application of Raman spectroscopy to industrial membranes. Part 2-Perfluorosulphonic membrane. Journal of Raman spectroscopy, 1991. 22(5): p. 275-279.

62. Liu, J.-n., Z.-r. Chen, and S.-F. Yuan, Study on the prediction of visible absorption maxima of azobenzene compounds. Journal of Zhejiang University. Science. B, 2005. 6(6): p. 584.

63. Khan, S., et al., Probing 2-acetylbenzofuran hydrazones and their metal complexes as $\alpha$ glucosidase inhibitors. Bioorganic Chemistry, 2020. 102: p. 104082.

64. Muthu, S. and J.U. Maheswari, Quantum mechanical study and spectroscopic (FT-IR, FT-Raman, 13C, 1H, UV) study, first order hyperpolarizability, NBO analysis, HOMO and LUMO analysis of 4-[(4-aminobenzene) sulfonyl] aniline by ab initio HF and density functional method. Spectrochimica Acta Part A: Molecular and Biomolecular Spectroscopy, 2012. 92: p. 154-163.

65. Govindarasu, K. and E. Kavitha, Vibrational spectra, molecular structure, NBO, UV, NMR, first order hyperpolarizability, analysis of 4-Methoxy-4'-Nitrobiphenyl by density functional theory. Spectrochimica Acta Part A: Molecular and Biomolecular Spectroscopy, 2014. 122: p. 130-141.

66. Govindarasu, K., E. Kavitha, and N. Sundaraganesan, Synthesis, structural, spectral (FTIR, FT-Raman, UV, NMR), NBO and first order hyperpolarizability analysis of $N$ phenylbenzenesulfonamide by density functional theory. Spectrochimica Acta Part A: Molecular and Biomolecular Spectroscopy, 2014. 133: p. 417-431. 
67. Govindarasu, K. and E. Kavitha, Molecular structure, vibrational spectra, NBO, UV and first order hyperpolarizability, analysis of 4-Chloro-dl-phenylalanine by density functional theory. Spectrochimica Acta Part A: Molecular and Biomolecular Spectroscopy, 2014. 133: p. 799-810.

68. Kleinman, D., Nonlinear dielectric polarization in optical media. Physical Review, 1962. 126(6): p. 1977.

69. Shelton, D.P. and J.E. Rice, Measurements and calculations of the hyperpolarizabilities of atoms and small molecules in the gas phase. Chemical Reviews, 1994. 94(1): p. 3-29.

70. Kumar, V.K., et al., Vibrational assignment of the spectral data, molecular dipole moment, polarizability, first hyperpolarizability, HOMO-LUMO and thermodynamic properties of 5-nitoindan using DFT quantum chemical calculations. Spectrochimica Acta Part A: Molecular and Biomolecular Spectroscopy, 2014. 118: p. 663-671. 


\section{Supplementary Files}

This is a list of supplementary files associated with this preprint. Click to download.

- GA.docx

- Spplementarylnformation.docx 